\title{
Holomorphic orbispheres in elliptic curve quotients and Diophantine equations
}

\author{
Hansol Hong and Hyung-Seok Shin
}

\begin{abstract}
We compute quantum cohomology rings of elliptic $\mathbb{P}^{1}$ orbifolds via orbicurve counting. The main technique is the classification theorem which relates holomorphic orbicurves with certain orbifold coverings. The countings of orbicurves are related to the integer solutions of Diophantine equations. This reproduces the computation of Satake and Takahashi in the case of $\mathbb{P}_{3,3,3}^{1}$ via a different method.
\end{abstract}

\section{Introduction}

The theory of holomorphic curves has been a great tool for understanding the geometry of a symplectic manifold. The quantum cohomology ring plays an important role in Hamiltonian dynamics and mirror symmetry. Quantum cohomology counts $J$-holomorphic spheres inside a symplectic manifold which intersect three given (co)cycles. As the counting also includes constant holomorphic spheres, quantum cohomology deforms the classical cup product on the singular cohomology ring.

Later, Chen and Ruan [CR] defined the quantum cohomology for symplectic orbifolds. Their orbifold quantum cohomology ring captures stringy phenomena in the sense that it also includes twisted sectors of a given orbifold as well as the usual cocycles on the underlying space of the orbifold. In order to do this, one also allows the domain curves to have orbifold singularities. That is, one should count holomorphic orbispheres as well.

The main objects which we will study throughout this article are among the simplest types of such orbifolds, spheres with three cone points called the orbifold projective lines. The orbifold quantum cohomology of these spheres indeed has a richer structure than that of the ordinary smooth sphere, since one additionally considers the interaction among three singular points through $J$-holomorphic orbispheres as mentioned. We shall briefly review the orbifold quantum cohomology in Section 3.

The orbifold projective lines that are of interest in this article are those which admit elliptic curves as their manifold covers and have three singular points $z_{1}$, $z_{2}$, and $z_{3}$. There are three such orbifold projective lines, $\mathbb{P}_{3,3,3}^{1}, \mathbb{P}_{2,3,6}^{1}$, and $\mathbb{P}_{2,4,4}^{1}$,

Kyoto Journal of Mathematics, Vol. 56, No. 2 (2016), 197-242

DOI $10.1215 / 21562261-3478871$, (C) 2016 by Kyoto University

Received January 5, 2015. Revised January 19, 2015. Accepted January 28, 2015.

2010 Mathematics Subject Classification: Primary 53D45; Secondary 57R18, 11D45. 
where the subindices indicate the orders of the singularities at the three orbifold points $z_{i}$. We will call these elliptic orbifold projective lines. (We remark that there is in fact one more orbifold projective line which is a quotient of elliptic curves, that is, the orbifold projective line $\mathbb{P}_{2,2,2,2}^{1}$ with four $\mathbb{Z}_{2}$-singular points, which will not be considered in this article.)

Recently, Satake-Takahashi [ST] computed the full genus 0 Gromov-Witten potential for $\mathbb{P}_{3,3,3}^{1}$ and $\mathbb{P}_{2,2,2,2}^{1}$, making use of an algebraic argument (e.g., Witten-Dijkgraaf-Verlinde-Verlinde (WDVV in short) equations). And furthermore, Krawitz-Shen [KS] independently computed the potentials for $\mathbb{P}_{3,3,3}^{1}, \mathbb{P}_{2,3,6}^{1}$, and $\mathbb{P}_{2,4,4}^{1}$ even for all genera and proved the Landau-Ginzburg/Calabi-Yau (LG/CY) correspondences for these examples.

We would like to mention that our method is different from theirs. Namely, our purpose here is to reproduce the (small) quantum product terms of the potential by directly counting holomorphic orbispheres. For this we will classify all holomorphic orbispheres with three markings in Section 4.2. Interestingly, we find that these orbispheres have a one-to-one correspondence with the solutions of certain Diophantine equations depending on the lattice structures on the universal covers of orbifold projective lines constructed from the preimages of the three singular points. As a result, we will provide simple expressions for the quantum cup products (Sections 5 and 6), making use of the formula for the number of solutions of a quadratic Diophantine equation (Appendix A).

The main results of the article are as follows. First, the classification of holomorphic orbispheres for the quantum product on $\mathbb{P}_{3,3,3}^{1}$ is related to the Diophantine equation $Q_{F}(a, b):=a^{2}-a b+b^{2}=d$. After fixing the first insertion to be $\Delta_{1}^{1 / 3}$, we will show that the moduli space of holomorphic orbispheres with three orbimarkings can be identified with the $\mathbb{Z}_{3}$-quotient of the space

$$
L(\mathbb{C}):=\{z \mapsto \lambda z \mid \lambda=a+b \tau(a, b \in \mathbb{Z})\} \quad\left(\tau=e^{2 \pi i / 3}\right),
$$

where the $\mathbb{Z}_{3}$-action on $L(\mathbb{C})$ is induced by multiplying $\lambda$ by $\tau$. Then, the above identification can be further decomposed into three pieces as

$$
\begin{aligned}
{\left[L(\mathbb{C}) / \mathbb{Z}_{3}\right] \cong } & (\text { i }) \mathcal{M}_{0,3}\left(\mathbb{P}_{3,3,3}^{1} ; \Delta_{1}^{1 / 3}, \Delta_{1}^{1 / 3}, \Delta_{1}^{1 / 3}\right) \\
& \cup(\text { ii })\left(\mathcal{M}_{0,3}\left(\mathbb{P}_{3,3,3}^{1} ; \Delta_{1}^{1 / 3}, \Delta_{2}^{1 / 3}, \Delta_{3}^{1 / 3}\right)\right. \\
& \left.\cup \mathcal{M}_{0,3}\left(\mathbb{P}_{3,3,3}^{1} ; \Delta_{1}^{1 / 3}, \Delta_{3}^{1 / 3}, \Delta_{2}^{1 / 3}\right)\right)
\end{aligned}
$$

depending on whether $(\mathrm{i}) Q_{F}(a, b) \equiv 0(\bmod 3)$ or $\left(\right.$ ii) $Q_{F}(a, b) \equiv 1(\bmod 3)$. We will also see that degree $d$ holomorphic spheres correspond to $\lambda=a+b \tau$ with $Q_{F}(a, b)=d$ (Sections 5.1 and 5.2). Finally, there is a $\mathbb{Z}_{6}$-action on $L(\mathbb{C})$ generated by $(1+\tau)$-multiplication, which in turn switches the two summands in (ii) (see Section 5.3). 


\section{THEOREM 1.1}

For $\mathbb{P}_{3,3,3}^{1}$, the only nontrivial 3-point Gromov-Witten invariants are

$$
\begin{gathered}
\left\langle\Delta_{1}^{1 / 3}, \Delta_{2}^{1 / 3}, \Delta_{3}^{1 / 3}\right\rangle_{0,3}^{\mathbb{P}_{3,3,3}^{1}}, \\
\left\langle\Delta_{i}^{1 / 3}, \Delta_{i}^{1 / 3}, \Delta_{i}^{1 / 3}\right\rangle_{0,3}^{\mathbb{P}_{3,3,3}^{1}}
\end{gathered}
$$

for $i=1,2,3$, where subindices of $\Delta$ indicate the singular points $z_{i}$. If one denotes the (compactified) moduli space of degree d holomorphic orbispheres contributing to (1.1) by $\overline{\mathcal{M}}_{0,3, d}\left(\mathbb{P}_{3,3,3}^{1} ; \Delta_{1}^{1 / 3}, \Delta_{1}^{1 / 3}, \Delta_{1}^{1 / 3}\right)$ and that for (1.2) by $\overline{\mathcal{M}}_{0,3, d}\left(\mathbb{P}_{3,3,3}^{1}\right.$; $\left.\Delta_{i}^{1 / 3}, \Delta_{i}^{1 / 3}, \Delta_{i}^{1 / 3}\right)$, then

$$
\begin{aligned}
& \# \overline{\mathcal{M}}_{0,3, d}\left(\mathbb{P}_{3,3,3}^{1} ; \Delta_{1}^{1 / 3}, \Delta_{2}^{1 / 3}, \Delta_{3}^{1 / 3}\right)=\frac{1}{6} \#\left\{(a, b): Q_{F}(a, b)=d, d \equiv 1(\bmod 3)\right\}, \\
& \# \overline{\mathcal{M}}_{0,3, d}\left(\mathbb{P}_{3,3,3}^{1} ; \Delta_{1}^{1 / 3}, \Delta_{1}^{1 / 3}, \Delta_{1}^{1 / 3}\right)=\frac{1}{3} \#\left\{(a, b): Q_{F}(a, b)=d, d \equiv 0(\bmod 3)\right\} .
\end{aligned}
$$

Similarly, solutions of the Diophantine equation $Q_{G}(a, b):=a^{2}+b^{2}=d$ are assigned to holomorphic orbispheres in $\mathbb{P}_{2,4,4}^{1}$, and the rest of the argument is parallel to that for $\mathbb{P}_{3,3,3}^{1}$.

\section{THEOREM 1.2}

For $\mathbb{P}_{2,4,4}^{1}$, the nontrivial contributions to 3-point Gromov-Witten invariants come only from the moduli spaces

$$
\begin{gathered}
\overline{\mathcal{M}}_{0,3, d}\left(\mathbb{P}_{2,4,4}^{1} ; \Delta_{1}^{1 / 2}, \Delta_{j}^{1 / 4}, \Delta_{k}^{1 / 4}\right), \quad \overline{\mathcal{M}}_{0,3, d}\left(\mathbb{P}_{2,4,4}^{1} ; \Delta_{j}^{2 / 4}, \Delta_{j}^{1 / 4}, \Delta_{j}^{1 / 4}\right), \\
\overline{\mathcal{M}}_{0,3, d}\left(\mathbb{P}_{2,4,4}^{1} ; \Delta_{j}^{2 / 4}, \Delta_{k}^{1 / 4}, \Delta_{k}^{1 / 4}\right)
\end{gathered}
$$

for $j, k=2,3$ and

$$
\begin{aligned}
& \# \overline{\mathcal{M}}_{0,3, d}\left(\mathbb{P}_{2,4,4}^{1} ; \Delta_{1}^{1 / 2}, \Delta_{j}^{1 / 4}, \Delta_{k}^{1 / 4}\right)=\frac{1}{4} \#\left\{(a, b): Q_{G}(a, b)=d, d \equiv 1(\bmod 4)\right\} \\
& \# \overline{\mathcal{M}}_{0,3, d}\left(\mathbb{P}_{2,4,4}^{1} ; \Delta_{j}^{2 / 4}, \Delta_{j}^{1 / 4}, \Delta_{j}^{1 / 4}\right)=\frac{1}{4} \#\left\{(a, b): Q_{G}(a, b)=d, d \equiv 0(\bmod 4)\right\} \\
& \# \overline{\mathcal{M}}_{0,3, d}\left(\mathbb{P}_{2,4,4}^{1} ; \Delta_{j}^{2 / 4}, \Delta_{k}^{1 / 4}, \Delta_{k}^{1 / 4}\right)=\frac{1}{4} \#\left\{(a, b): Q_{G}(a, b)=d, d \equiv 2(\bmod 4)\right\}
\end{aligned}
$$

The quantum product on $\mathbb{P}_{2,3,6}^{1}$ is also related to the Diophantine equation $Q_{F}(a, b):=a^{2}+b^{2}=d$, but now we consider $d$ modulo 6 .

\section{PROPOSITION 1.3}

For $\mathbb{P}_{2,3,6}^{1}$, we also have a similar statement related to the number of solutions of $Q_{F}(a, b)=d$ considering $d(\bmod 6)$ and $d(\bmod 3)$. Nontrivial 3 -point GromovWitten invariants are listed as follows:

$$
\# \overline{\mathcal{M}}_{0,3, d}\left(\mathbb{P}_{2,3,6}^{1} ; \Delta_{1}^{1 / 2}, \Delta_{2}^{1 / 3}, \Delta_{3}^{1 / 6}\right)=\frac{1}{6} \#\left\{(a, b): Q_{F}(a, b)=d, d \equiv 1(\bmod 6)\right\}
$$




$$
\begin{aligned}
& \# \overline{\mathcal{M}}_{0,3, d}\left(\mathbb{P}_{2,3,6}^{1} ; \Delta_{3}^{3 / 6}, \Delta_{2}^{1 / 3}, \Delta_{3}^{1 / 6}\right)=\frac{1}{6} \#\left\{(a, b): Q_{F}(a, b)=d, d \equiv 4(\bmod 6)\right\} \\
& \# \overline{\mathcal{M}}_{0,3, d}\left(\mathbb{P}_{2,3,6}^{1} ; \Delta_{1}^{1 / 2}, \Delta_{3}^{2 / 6}, \Delta_{3}^{1 / 6}\right)=\frac{1}{6} \#\left\{(a, b): Q_{F}(a, b)=d, d \equiv 3(\bmod 6)\right\} \\
& \# \overline{\mathcal{M}}_{0,3, d}\left(\mathbb{P}_{2,3,6}^{1} ; \Delta_{3}^{3 / 6}, \Delta_{3}^{2 / 6}, \Delta_{3}^{1 / 6}\right)=\frac{1}{6} \#\left\{(a, b): Q_{F}(a, b)=d, d \equiv 0(\bmod 6)\right\}
\end{aligned}
$$

$$
\begin{aligned}
& \# \overline{\mathcal{M}}_{0,3,2 d}\left(\mathbb{P}_{2,3,6}^{1} ; \Delta_{3}^{2 / 6}, \Delta_{3}^{2 / 6}, \Delta_{3}^{2 / 6}\right)=\frac{1}{6} \#\left\{(a, b): Q_{F}(a, b)=d, d \equiv 0(\bmod 3)\right\}, \\
& \# \overline{\mathcal{M}}_{0,3,2 d}\left(\mathbb{P}_{2,3,6}^{1} ; \Delta_{2}^{1 / 3}, \Delta_{2}^{1 / 3}, \Delta_{3}^{2 / 6}\right)=\frac{1}{6} \#\left\{(a, b): Q_{F}(a, b)=d, d \equiv 1(\bmod 3)\right\}, \\
& \# \overline{\mathcal{M}}_{0,3,2 d}\left(\mathbb{P}_{2,3,6}^{1} ; \Delta_{2}^{1 / 3}, \Delta_{2}^{1 / 3}, \Delta_{2}^{1 / 3}\right)=\frac{1}{3} \#\left\{(a, b): Q_{F}(a, b)=d, d \equiv 0(\bmod 3)\right\} .
\end{aligned}
$$

In item (2), there are only even-degree holomorphic orbispheres.

In addition to these, there are two more 3-point Gromov-Witten invariants $\left\langle\Delta_{3}^{1 / 6}, \Delta_{3}^{1 / 6}, \Delta_{3}^{4 / 6}\right\rangle_{0,3}^{\mathbb{P}_{2,3,6}^{1}}$ and $\left\langle\Delta_{2}^{2 / 3}, \Delta_{3}^{1 / 6}, \Delta_{3}^{1 / 6}\right\rangle_{0,3}^{\mathbb{P}_{2,3,6}^{1}}$, for which we only give a heuristic counting in Conjecture 6.3. We are not able to associate solutions of a Diophantine equation to these holomorphic orbispheres since their domain is $\mathbb{P}_{3,6,6}^{1}$, which does not admit an elliptic curve as a covering unlike the other cases.

On the other hand, closed-string mirror symmetry for $\mathbb{P}_{a, b, c}^{1}$ has been intensively studied in many preceding works. For example, Gromov-Witten theory on $\mathbb{P}_{a, b, c}^{1}$ with $\frac{1}{a}+\frac{1}{b}+\frac{1}{c}>1$ was investigated by Milanov-Tseng [MT] and Rossi [R] using Frobenius structures associated with the mirror potential $W$ for $\mathbb{P}_{a, b, c}^{1}$. For elliptic orbifold projective lines, global mirror symmetry and LG/CY correspondence were proved by Milanov-Ruan [MR], Krawitz-Shen [KS], and MilanovShen [MSh].

In the mirror symmetry point of view, quantum cohomology rings of elliptic projective lines should be isomorphic to the Jacobian rings of their mirror potentials:

$$
\mathfrak{k}_{\mathfrak{s}}: Q H_{\text {orb }}^{*}\left(\mathbb{P}_{3,3,3}^{1}\right) \rightarrow \operatorname{Jac}\left(W_{3,3,3}\right) .
$$

We remark that $W_{a, b, c}$ has been explicitly computed in [CHKL] and the computation in this article should be helpful to understand such a mirror symmetry isomorphism.

\section{Preliminaries}

In this section, we briefly review orbifold theory which will be used in Section 4. 


\subsection{Orbifolds}

The notion of orbifolds was first introduced by Satake [Sa] under the name $V$ manifolds, and the theory of orbifolds was further developed by Thurston [Thu]. Here we follow the definition of orbifold as given in [CR1] (see [ALR] for more details on other approaches).

\section{DEFINITION 2.1}

A paracompact Hausdorff space $X$ is called an $n$-dimensional orbifold if $X$ can be covered by open subsets $X=\bigcup U_{i}$ such that

(1) there exists a homeomorphism $\phi_{i}: V_{i} / G_{i} \rightarrow U_{i}$ where $V_{i}$ is an open subset of $\mathbb{R}^{n}$ with an (effective) action of a finite group $G_{i}$;

(2) for a point $x \in U_{i} \cap U_{j}$, there is an open neighborhood $U_{i j}$ of $x$ in $X$ which is isomorphic to the quotient of an open domain $V_{i j}$ in $\mathbb{R}^{n}$ by a finite group $G_{i j}$, and there are embeddings $\left(V_{i j} \rightarrow V_{i}, G_{i j} \rightarrow G_{i}\right)$ and $\left(V_{i j} \rightarrow V_{j}, G_{i j} \rightarrow G_{j}\right)$ which are equivariant.

The local model $\left(V_{i}, G_{i}, \phi_{i}\right)$ of $X$ is called a local uniformizing chart. We denote by $|X|$ the underlying topological space obtained by forgetting the orbifold structure of $X$.

In particular, for each $x \in X$, we can take an open neighborhood $U_{x}$ of $x$ in $X$ which is isomorphic to $V_{x} / G_{x}$ (where $V_{x} \subset \mathbb{R}^{n}$ and $G_{x}$ is a finite group) such that the preimage of $x$ in $V_{x}$ is the single point which is fixed by $G_{x}$. We call $\left(V_{x}, G_{x}\right)$ a local uniformizing chart around $x$. Now, for two orbifolds $X$ and $Y$, the morphism between them can be defined as follows.

\section{DEFINITION 2.2}

(1) A smooth map $f$ between $X$ and $Y$ is a continuous map $f: X \rightarrow Y$ which has the following local property. For each $x \in X$, there exist uniformizing charts $\left(V_{x}, G_{x}\right)$ and $\left(V_{f(x)}, G_{f(x)}\right)$ of $x$ and $f(x)$, respectively, and an injective group homomorphism $G_{x} \rightarrow G_{f(x)}$ such that $f$ admits a local smooth lifting $\tilde{f}_{V_{x} V_{f(x)}}$ : $V_{x} \rightarrow V_{f(x)}$ which is $\left(G_{x}, G_{f(x)}\right)$-equivariant.

(2) A smooth map $f$ between $X$ and $Y$ is called good if it admits a collection of maps $\left\{\widetilde{f}_{U U^{\prime}}, \lambda\right\}$ which is called a compatible system of $f$ and is defined as follows. For each equivariant embedding $i:\left(V_{2}, G_{2}, \pi_{2}\right) \rightarrow\left(V_{1}, G_{1}, \pi_{1}\right)$ of local uniformizing charts of $X$, there is an equivariant embedding $\lambda(i):\left(V_{2}^{\prime}, G_{2}^{\prime}, \pi_{2}^{\prime}\right) \rightarrow$ 
$\left(V_{1}^{\prime}, G_{1}^{\prime}, \pi_{1}^{\prime}\right)$ of charts of $Y$ with the following commuting diagram:

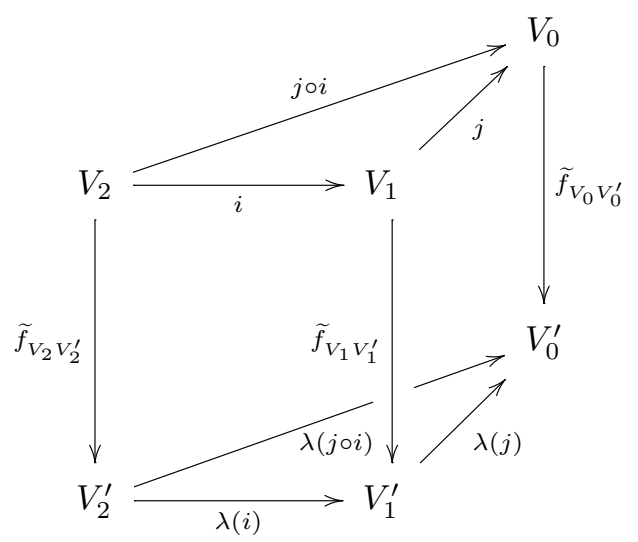

where each map is an equivariant map.

There is another notion of maps between orbifolds introduced by Takeuchi [T]. We will compare the two notions in Lemma 2.10.

\subsection{Orbifold fundamental group}

In this section, we recall the notion of the orbifold fundamental group (introduced by Thurston), which is closely related to the orbifold covering theory explained below. This is analogous to the connection between the usual covering theory and fundamental groups. It is enough to consider global quotient orbifolds for our purpose in this article. Consider a finite group action $G$ on a manifold $M$ which preserves orientation. (One may consider an infinite group $G$ if an orbifold is represented as a quotient of noncompact manifolds by a locally free action of a discrete group.) Then, generalized loops in $M$ are defined as follows.

\section{DEFINITION 2.3}

A path $\gamma:[0,1] \rightarrow M$ is called a generalized loop based at $\widetilde{x_{0}} \in M$ if $\gamma(0)=\widetilde{x_{0}}$ and there exists $g_{\gamma} \in G$ such that $\gamma(1)=g \cdot \gamma(0)$.

Choose a point $\widetilde{x_{0}} \in M$ with $G_{\widetilde{x_{0}}}=1$, and let $\pi_{1}^{\text {orb }}([M / G])$ be the set of equivalence classes of generalized loops based at $\widetilde{x_{0}}$ where the equivalence relation is given as homotopies fixing endpoints. One can check that $\pi_{1}^{\text {orb }}([M / G])$ has a natural group structure by defining

$$
[\gamma] \cdot[\delta]=\left[\gamma \# g_{\gamma}(\delta)\right]
$$

for generalized loops $\gamma, \delta$ based at $\widetilde{x_{0}}$ where \# denotes the concatenation of paths.

For example, $\pi_{1}^{\text {orb }}\left(\mathbb{P}_{a, b, c}^{1}\right)$ has a presentation

$$
\pi_{1}^{\text {orb }}\left(\mathbb{P}_{a, b, c}\right)=\left\langle\rho_{1}, \rho_{2}, \rho_{3} \mid\left(\rho_{1}\right)^{a}=\left(\rho_{2}\right)^{b}=\left(\rho_{3}\right)^{c}=\rho_{1} \rho_{2} \rho_{3}=1\right\rangle,
$$

where $\rho_{1}, \rho_{2}$, and $\rho_{3}$ are generalized loops in the universal cover of $\mathbb{P}_{a, b, c}^{1}$ whose images in the underlying space of the orbifold look as in Figure 1(a). The relation 


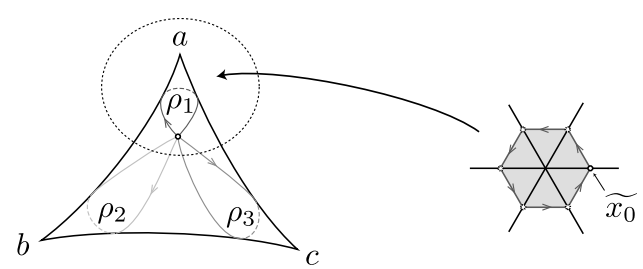

(a)

(b)

Figure 1. (a) Generators of $\pi_{1}^{\text {orb }}\left(\mathbb{P}_{a, b, c}^{1}\right)$ and (b) the relation $\rho_{1}^{a}=1$.

$\rho_{1}^{a}=1$ can be seen in the uniformizing chart around the singular point of order $a$ (see Figure $1(\mathrm{~b})$ ). One can observe the relation $\rho_{1} \rho_{2} \rho_{3}=1$ even more directly on the orbifold itself.

\section{REMARK 2.4}

See [T] for details on the link between orbifold fundamental groups and orbifold coverings which we shall explain below.

\subsection{Orbifold covering theory}

There is an analogue of covering space for orbifolds whose local model is $\mathbb{R}^{n} / G^{\prime} \rightarrow$ $\mathbb{R}^{n} / G$ for some finite group $G$ which acts on $\mathbb{R}^{n}$ with $G^{\prime} \leq G$.

\section{DEFINITION 2.5 ([T, SECTION 1])}

An orbifold $\widetilde{X}$ is called a covering orbifold if there is a continuous surjective map $p:|\widetilde{X}| \rightarrow|X|$ satisfying the following condition. For each point $x \in|X|$, there is a local uniformizing chart $\widetilde{U}_{x} / G_{x} \cong U_{x}$ such that each point $\tilde{x} \in p^{-1}(x)$ has a local uniformizing chart $\widetilde{U}_{x} / G_{x, i} \cong V_{x, i}$ for some $G_{x, i} \leq G_{x}$ such that the following diagram commutes:

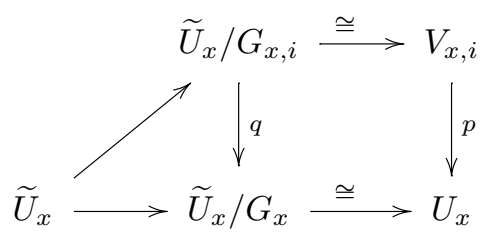

where $q$ is the natural projection.

An orbifold which admits a manifold covering is called a good orbifold. For example, the orbifold projective lines $\mathbb{P}_{3,3,3}^{1}, \mathbb{P}_{2,4,4}^{1}$, and $\mathbb{P}_{2,3,6}^{1}$ that are our main concern are all good orbifolds, as they are given by quotients of a 2-torus. Throughout the section, we assume that all orbifolds are good.

Following $[\mathrm{T}]$ we introduce the notion of an orbimap. 
DEFINITION 2.6 ([T, SECTION 2])

An orbimap $f: X \rightarrow Y$ consists of a continuous map $h:|X| \rightarrow|Y|$ between underlying spaces and a fixed continuous map $\tilde{h}: \widetilde{X} \rightarrow \widetilde{Y}$ which satisfy

(1) $h \circ p=q \circ \tilde{h}$;

(2) for each $\sigma \in \operatorname{Aut}(\widetilde{X}, p)\left(\cong \pi_{1}^{\text {orb }}(X)\right)$, there exists $\tau \in \operatorname{Aut}(\tilde{Y}, q)$ such that $\tilde{h} \circ \sigma=\tau \circ \tilde{h} ;$

(3) $h(|X|)$ does not lie in the singular loci of $Y$ entirely.

\section{REMARK 2.7}

Indeed, the covering theory in [T] only concerns good orbifolds.

For orbimaps, we have the usual lifting theorems in covering theory as well, the proofs of which are not very much different from the standard one.

PROPOSITION 2.8 ([T, PROPOSITION 2.7])

Let $f:(X, x) \rightarrow(Y, y)$ be an orbimap, and let $p:\left(Y^{\prime}, y^{\prime}\right) \rightarrow(Y, y)$ be a covering. Then $f$ can be lifted to an orbimap $\tilde{f}: X \rightarrow Y^{\prime}$ if and only if $f_{*} \pi_{1}^{\text {orb }}(X, x) \subset$ $p_{*} \pi_{1}^{\text {orb }}\left(Y^{\prime}, y^{\prime}\right)$.

As we will often use lifting theorems for orbifold coverings by Takeuchi [T], we also introduce another notion of morphisms between orbifolds which Takeuchi called orbimaps. However, we will show in Section 2.4 that the two notions are equivalent for our main examples.

\subsection{Orbimaps between 2-dimensional orbifolds}

While orbifold covering theory was well established for orbimaps by Takeuchi [T], orbifold quantum cohomology is defined by counting the good maps given in Definition 2.2(2). In the case of elliptic $\mathbb{P}^{1}$-orbifolds, it will be shown that the maps given in Definition 2.2(1) satisfy the axioms in Definition 2.6.

\section{REMARK 2.9}

From [CR, Lemma 4.4.11], a smooth map $f: X \rightarrow X^{\prime}$ between two orbifolds $X$ and $X^{\prime}$ is a good map with a unique compatible system up to isomorphism if the inverse image of the regular part of $X^{\prime}$ is an open, dense, and connected subset of $X$. Note that a nonconstant smooth map contributing to the quantum product for $\mathbb{P}_{a, b, c}^{1}$ automatically satisfies this property.

Consider two (good) orbifolds $X$ and $Y$ which admit manifold universal covering spaces $p: \widetilde{X} \rightarrow X$ and $q: \widetilde{Y} \rightarrow Y$, respectively. Assume that the deck transformation actions of $p$ and $q$ are orientation preserving. Moreover, assume that both $X$ and $Y$ are 2-dimensional (which is the case of our main interest). Note that singular loci of $X$ and $Y$ are sets of isolated points. 
LEMMA 2.10

With the setting as above, any nonconstant smooth map $\phi: X \rightarrow Y$ satisfies the axioms in Definition 2.6 if $\operatorname{dim} X=\operatorname{dim} Y=2$.

Proof

We first show that there is a continuous map $\tilde{\phi}: \tilde{X} \rightarrow \tilde{Y}$ which lifts $\phi: X \rightarrow Y$.

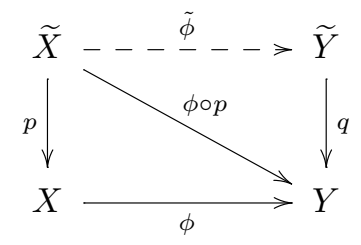

What we want to have is basically a lift of the map $\phi \circ p: \widetilde{X} \rightarrow Y$. We claim that at each point $\tilde{x} \in \tilde{X}$ there is a local lifting of $\phi \circ p$. Let $x:=p(\tilde{x})$. Then one can find a neighborhood of $\tilde{x}$ which uniformizes $X$ locally around $x$. Since the same is true for any point in the inverse image $q^{-1}(\phi(x))$, we can find a local lifting of $\phi$ around $\tilde{x}$ by the properties of orbifold maps.

By gathering such a neighborhood for each $\tilde{x} \in \tilde{X}$, we obtain an open covering $\widetilde{\mathcal{U}}=\left\{\widetilde{U}_{i}: \widetilde{U}_{i} \subset \widetilde{X}, i \in I\right\}$ of $\widetilde{X}$ which consists of open subsets of $\widetilde{X}$ on which $\phi$ can be locally lifted. For each $\widetilde{U}_{i} \in \tilde{\mathcal{U}}$, we fix a local lifting $\tilde{\phi}_{i}$ of $\phi$. On the intersection of two open subsets $\widetilde{U}_{i}$ and $\widetilde{U}_{j}$ in $\widetilde{\mathcal{U}}$, two local liftings $\tilde{\phi}_{i}$ and $\tilde{\phi}_{j}$ differ by an element $g_{i j}$ of $\operatorname{Aut}(\widetilde{Y}, q) \cong \pi_{1}^{\text {orb }}(Y)$, that is,

$$
\left.\tilde{\phi}_{i}\right|_{\widetilde{U}_{i} \cap \widetilde{U}_{j}}=\left.g_{i j} \circ \tilde{\phi}_{j}\right|_{\widetilde{U}_{i} \cap \widetilde{U}_{j}} \text {. }
$$

Note that $\left\{g_{i j}: i, j \in I\right\}$ satisfies the usual cocycle condition; that is,

$$
g_{i j} g_{j k} g_{k i}=1 \text {. }
$$

We obtain (2.3) from

$$
\begin{aligned}
\tilde{\phi}_{i} & =g_{i j} \circ \tilde{\phi}_{j} \\
& =\left(g_{i j} g_{j k}\right) \circ \tilde{\phi}_{k} \\
& =\left(g_{i j} g_{j k} g_{k i}\right) \circ \tilde{\phi}_{i}
\end{aligned}
$$

on $\widetilde{U}_{i} \cap \widetilde{U}_{j} \cap \widetilde{U}_{k}$ and the fact that the action of $\pi_{1}^{\text {orb }}(Y)$ on $Y$ is free generically. (Recall that $\phi$ is a nonconstant map.)

Therefore, $\left\{g_{i j}\right\}_{i, j \in I}$ defines a principal $\pi_{1}^{\text {orb }}(Y)$-bundle over $\widetilde{X}$ or equivalently a covering space of $\widetilde{X}$. Here, $g_{i j}$ glues $\widetilde{U}_{i} \times \pi_{1}^{\text {orb }}(Y)$ and $\widetilde{U}_{j} \times \pi_{1}^{\text {orb }}(Y)$ by left multiplication so that the resulting bundle admits the right action of $\pi_{1}^{\text {orb }}(Y)$. Since $\pi_{1}^{\text {orb }}(Y)$ is discrete and $\widetilde{X}$ is simply connected, this bundle should be trivial. Therefore, the cocycle $\left\{g_{i j}\right\}$ is also trivial up to coboundary, that is, there exists a collection $\left\{\left(\epsilon_{i}, \widetilde{U}_{i}\right) \in \pi_{1}^{\text {orb }}(Y) \times \widetilde{\mathcal{U}}: i \in I\right\}$ of elements of $\pi_{1}^{\text {orb }}(Y)$ each of which is associated with an open subset in $\widetilde{\mathcal{U}}$ such that

$$
g_{i j}=\epsilon_{i}^{-1} \epsilon_{j}
$$


(In other words, $\left\{\epsilon_{i}\right\}$ trivializes the principal bundle corresponding to the data $\left.\left\{g_{i j}\right\}.\right)$

From (2.2) and (2.4), we have

$$
\epsilon_{i} \tilde{\phi}_{i}=\epsilon_{j} \tilde{\phi}_{j}
$$

on $\widetilde{U}_{i} \cap \widetilde{U}_{j}$. If we set $\tilde{\phi}_{i}^{\prime}:=\epsilon_{i} \tilde{\phi}_{i}$, then $\left\{\tilde{\phi}_{i}^{\prime}\right\}_{i \in I}$ gives a collection of local liftings of $\phi$, any two of which agree on their common domain. Denote the resulting global lifting of $\phi$ by $\tilde{h}$.

We next check the second axiom of Definition 2.6. Let $\sigma$ be a deck transformation of the covering $p: \widetilde{X} \rightarrow X$, that is, an element of $\operatorname{Aut}(\widetilde{X}, p)$. Then $\tilde{h} \circ \sigma$ is a lifting of $\phi \circ p$ because

$$
\begin{aligned}
q \circ \tilde{h} \circ \sigma & =(\phi \circ p) \circ \sigma \\
& =\phi \circ(p \circ \sigma) \\
& =\phi \circ p .
\end{aligned}
$$

Since both $\tilde{h}$ and $\tilde{h} \circ \sigma$ are liftings of $\phi \circ p$, one can find an element $\tau_{\tilde{x}}$ in $\operatorname{Aut}(Y, q)$ for each $\tilde{x} \in \widetilde{X}$ such that

$$
\tilde{h} \circ \sigma(\tilde{x})=\tau_{\tilde{x}}(\tilde{h}(\tilde{x})) .
$$

Since $\widetilde{X}$ is connected and $\pi_{1}^{\text {orb }}(Y)$ is discrete, $\tau_{\tilde{x}}$ has to be independent of $\tilde{x}$. This gives an element $\tau$ that holds for the second property of orbimaps.

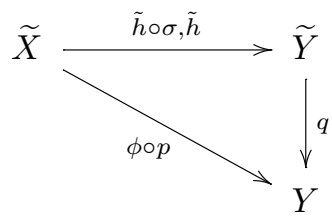

Finally, the third condition of orbimaps follows obviously since we are only considering nonconstant morphisms between orbifolds with the same dimension.

\section{REMARK 2.11}

From the proof, we see that the lemma also holds for a smooth map between two good orbifolds of general dimension which does not send a whole open subset to a fixed locus.

\section{Gromov-Witten theory of orbifolds}

In this section, we briefly review the quantum cohomology of orbifolds developed by Chen and Ruan. The key ingredients in defining the product on this cohomology are holomorphic orbispheres (or orbifold stable maps in general) in orbifolds with three marked points. If we consider such spheres with an arbitrary number of markings as well, then we obtain the orbifold (genus 0) Gromov-Witten invariants (see [CR]; see also Sections 3.1 and 3.2). 
At the end of the section, we will come back to our main examples, orbifold projective lines $\mathbb{P}_{a, b, c}^{1}$, and their Gromov-Witten theory. We are particularly interested in $\mathbb{P}_{a, b, c}^{1}$ with $\frac{1}{a}+\frac{1}{b}+\frac{1}{c}=1$, which are in fact quotients of an elliptic curve and exhibit lots of number-theoretic phenomena. We remark that Satake and Takahashi [ST] provided the full genus 0 potential of $\mathbb{P}_{3,3,3}^{1}$ using the algebraic method. We will also briefly recall their work.

\subsection{Description of $\overline{\mathcal{M}}_{g, k, \beta}(X)$}

Let $(X, \omega)$ be a compact effective symplectic orbifold with a compatible almost complex structure $J$ (see [CR, Definitions 2.1.1, 2.1.5]). We begin with the description of the compactified moduli space of orbifold stable maps into $X$. The details can be found in [CR].

\section{DEFINITION 3.1 ([CR, DEFINITION 2.2.2])}

An orbi-Riemann surface of genus $g$ is a triple $(\Sigma, \boldsymbol{z}, \boldsymbol{m})$ where

- $\Sigma$ is a smooth Riemann surface of genus $g$, and

- $\boldsymbol{z}=\left(z_{1}, \ldots, z_{k}\right)$ is a set of orbisingular points on $\Sigma$ with isotropy group of order $\boldsymbol{m}=\left(m_{1}, \ldots, m_{k}\right)$ for some integer $m_{i} \geq 2$. The orbifold structure on $\Sigma$ is given as follows: at each point $z_{i}$, a disc neighborhood of $z_{i}$ is uniformized by the branched covering map $z \rightarrow z^{m_{i}}$.

In order to compactify the moduli space, we should also include nodal Riemann surfaces as domains of holomorphic maps.

\section{DEFINITION 3.2 ([CR, DEFINITION 2.3.1])}

A nodal Riemann surface with $k$ marked points is a pair $(\Sigma, \boldsymbol{z})$ of a connected topological space $\Sigma=\bigcup \pi_{\Sigma_{\nu}}\left(\Sigma_{\nu}\right)$ and a set of $k$ distinct points $\boldsymbol{z}=\left(z_{1}, \ldots, z_{k}\right)$ in $\Sigma$ with the following properties.

- $\Sigma_{\nu}$ is a smooth Riemann surface of genus $g_{\nu}$, and $\pi_{\nu}: \Sigma_{\nu} \rightarrow \Sigma$ is a continuous map. The number of components $\Sigma_{\nu}$ is finite.

- For each $z \in \Sigma_{\nu}$, there is a neighborhood around it such that the restriction of $\pi_{\nu}: \Sigma_{\nu} \rightarrow \Sigma$ to this neighborhood is a homeomorphism to its image.

- For each $z \in \Sigma$, we have $\sum_{\nu} \# \pi_{\nu}^{-1}(z) \leq 2$, and the set of nodal points $\left\{z \mid \sum_{\nu} \# \pi_{\nu}^{-1}(z)=2\right\}$ is finite.

- $z$ does not contain any nodal points.

We next allow cone singularities on nodal Riemann surfaces.

DEFINITION 3.3 ([CR, DEFINITION 2.3.2])

A nodal orbi-Riemann surface is a nodal marked Riemann surface $(\Sigma, \boldsymbol{z})$ with an orbifold structure as follows. 
- The set of orbisingular points is contained in the set of marked points and nodal points $\boldsymbol{z}$.

- A disk neighborhood of a marked point is uniformized by a branched covering map $z \rightarrow z^{m_{i}}$.

- A neighborhood of a nodal point is uniformized by the chart $\left(\widetilde{U}, \mathbb{Z}_{n_{j}}\right)$, where $\widetilde{U}=\left\{(x, y) \in \mathbb{C}^{2} \mid x y=0\right\}$ on which $\mathbb{Z}_{n_{j}}$ acts by $e^{\frac{2 \pi i}{n_{j}}} \cdot(x, y)=\left(e^{\frac{2 \pi i}{n_{j}}} x, e^{-\frac{2 \pi i}{n_{j}}} y\right)$.

Here $m_{i}$ and $n_{j}$ are allowed to be 1 . We denote the corresponding nodal orbiRiemann surface by $(\Sigma, \boldsymbol{z}, \boldsymbol{m}, \boldsymbol{n})$, and if there is no confusion, then we simply write it as $(\Sigma, \boldsymbol{z})$ (see Figure 2).

With a nodal orbi-Riemann surface as the domain, an orbifold stable map is defined as follows.

\section{DEFINITION 3.4 ([CR, DEFINITION 2.3.3])}

For a given almost complex orbifold $(X, J)$, an orbifold stable map is a triple $(f,(\Sigma, \boldsymbol{z}, \boldsymbol{m}, \boldsymbol{n}), \xi)$ with the following properties.

- $f: \Sigma \rightarrow|X|$ is a continuous map from a nodal Riemann surface $\Sigma$ such that $f_{\nu}=f \circ \pi_{\nu}$ is a $J$-holomorphic map.

- Consider the local lifting $\widetilde{f}_{V_{x} V_{f(x)}}:\left(V_{x}, G_{x}\right) \rightarrow\left(V_{f(x)}, G_{f(x)}\right)$ of $f$. The homomorphism $G_{x} \rightarrow G_{f(x)}$ is injective.

- Let $k_{\nu}$ be the number of points in $\Sigma_{\nu}$ which are marked or nodal. If $f_{\nu}$ is a constant map, then $k_{\nu}+2 g_{\nu} \geq 3$.

- $\xi$ is an isomorphism class of compatible systems.

For the definition of an isomorphism between compatible systems, see [CR, Definition 4.4.4].

We are now ready to define the moduli space relevant to the orbifold GromovWitten invariants of $X$.

\section{DEFINITION 3.5}

(1) Two stable maps $(f,(\Sigma, \boldsymbol{z}), \xi)$ and $\left(f^{\prime},\left(\Sigma^{\prime}, \boldsymbol{z}^{\prime}\right), \xi^{\prime}\right)$ are equivalent if there is an isomorphism $\theta:(\Sigma, \boldsymbol{z}, \boldsymbol{m}, \boldsymbol{n}) \rightarrow\left(\Sigma^{\prime}, \boldsymbol{z}^{\prime}, \boldsymbol{m}^{\prime}, \boldsymbol{n}^{\prime}\right)$ such that $f^{\prime} \circ \theta=f$ and $\xi^{\prime} \circ$ $\theta=\xi$.

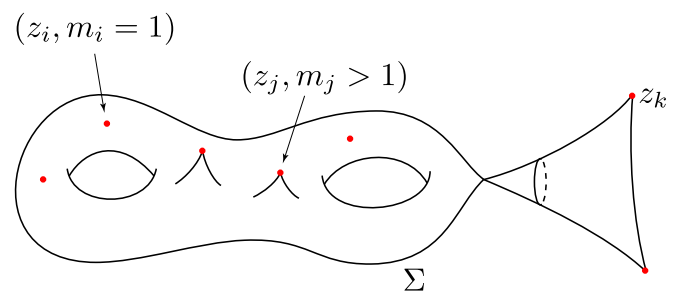

Figure 2. A nodal orbi-Riemann surface. 
(2) Given a homology class $\beta \in H_{2}(|X| ; \mathbb{Z}), \overline{\mathcal{M}}_{g, k, \beta}(X, J)$ is defined as the moduli space of equivalence classes of orbifold stable maps of genus $g$, with $k$ marked points, and of homology class $\beta$.

\subsection{Gromov-Witten invariants of orbifolds}

Now, we recall the definition of the orbifold cohomology group $H_{\text {orb }}^{*}(X, \mathbb{Q})$ from [CR1] which is freely generated by the elements of the cohomology groups of twisted sectors of $X$ (i.e., $H_{\text {orb }}^{*}(X, \mathbb{Q})=H^{*}(I X, \mathbb{Q})$ as a vector space, where $I X$ is the inertia orbifold of $X)$. Here, the degrees of elements in $H^{*}\left(X_{(g)}, \mathbb{Q}\right)$ are shifted by $2 \iota(g)$ where $X_{(g)}$ is the twisted sector associated with the conjugacy class $(g)$ and $\iota(g)$ is the age of an element $g$ in a local group. Also, $H_{\text {orb }}^{*}(X, \mathbb{Q})$ admits a natural Poincaré pairing which is compatible with these shifted degrees:

$$
\int_{I X}^{\text {orb }}(\cdot) \cup_{\text {orb }}(\cdot): H_{\text {orb }}^{*}(X, \mathbb{Q}) \otimes H_{\text {orb }}^{*}(X, \mathbb{Q}) \rightarrow \mathbb{Q} .
$$

We fix a $\mathbb{Q}$-basis $\left\{\gamma_{i}\right\}_{i=1, \ldots, N}$ of $H_{\text {orb }}^{*}(X, \mathbb{Q})$. Then the $k$-point GromovWitten invariants are defined by the following equation:

$$
\left\langle\gamma_{1}, \ldots, \gamma_{k}\right\rangle_{g, k, \beta}^{X}:=\int_{\left[\overline{\mathcal{M}}_{0, k, \beta}(X)\right]^{\mathrm{vir}}} e v_{1}^{*} \gamma_{1} \wedge \cdots \wedge e v_{k}^{*} \gamma_{k} .
$$

We also define $\left\langle\gamma_{1}, \ldots, \gamma_{k}\right\rangle_{g, k}^{X}$ to be the weighted sum $\sum_{\beta}\left\langle\gamma_{1}, \ldots, \gamma_{k}\right\rangle_{g, k, \beta}^{X} q^{\omega(\beta)}$.

\section{REMARK 3.6}

The compactified moduli space $\overline{\mathcal{M}}_{0, k, \beta}(X)$ admits a virtual fundamental class $\left[\overline{\mathcal{M}}_{0, k, \beta}(X)\right]^{\mathrm{vir}}$ which can be defined with the help of an abstract perturbation technique in general. (Readers are referred to [CR] for more details.)

For a tuple $\boldsymbol{x}=\left(X_{\left(g_{1}\right)}, X_{\left(g_{2}\right)}, \ldots, X_{\left(g_{k}\right)}\right)$ of twisted sectors, we say that $(f,(\Sigma, \boldsymbol{z}), \xi)$ is of type $\boldsymbol{x}$ if orbi-insertions at the marked point $z_{i}$ lie in $H^{*}\left(X_{\left(g_{i}\right)}, \mathbb{Q}\right)$ for each $i$. Let $\overline{\mathcal{M}}_{g, k, \beta}(X, J, \beta, \boldsymbol{x})$ denote the moduli space of equivalence classes of orbifold stable maps of genus $g$ with $k$ marked points and of homology class $\beta$ and type $\boldsymbol{x}$. Then $\overline{\mathcal{M}}_{0, k, \beta}(X)$ is the union of $\overline{\mathcal{M}}_{g, k, \beta}(X, J, \beta, \boldsymbol{x})$ over all types $\boldsymbol{x}$, and the integration in (3.2) is nonzero on components $\overline{\mathcal{M}}_{g, k, \beta}(X, J, \beta, \boldsymbol{x})$ with $\gamma_{i} \in H^{*}\left(X_{\left(g_{i}\right)}, \mathbb{Q}\right)$.

For later purposes, we give the virtual dimension of $\overline{\mathcal{M}}_{g, k, \beta}(X, J, \beta, \boldsymbol{x})$ explicitly as

$$
2 c_{1}(T X)(\beta)+2(n-3)(1-g)+2 k-2 \iota(\boldsymbol{x}),
$$

where $2 n=\operatorname{dim}_{\mathbb{R}} X$ and $\iota(\boldsymbol{x})=\sum_{i=1}^{k} \iota\left(g_{i}\right)$ (see [CR, Proposition 3.2.5]).

\section{REMARK 3.7}

If $c_{1}$ of $X$ vanishes as in our elliptic examples, then the virtual dimension of the moduli is independent of the homology class $\beta$. In particular, $n=1$ and $g=0$ in our main examples. 
If we set $\boldsymbol{t}:=\sum t_{i} \gamma_{i}$, then the generating function for the Gromov-Witten invariants is defined as

$$
F_{0}^{X}(\boldsymbol{t}):=\sum_{k, \beta} \frac{1}{k !}\langle\boldsymbol{t}, \ldots, \boldsymbol{t}\rangle_{0, k, \beta}^{X} q^{\omega(\beta)},
$$

which we will call the genus 0 Gromov-Witten potential for $X$.

In particular, when $k=3$, the counting given in (3.2) defines a product $*$ on $H_{\text {orb }}^{*}(X, \mathbb{Q})$ which is called the quantum product. More precisely,

$$
\int_{X}^{\mathrm{orb}}\left(\gamma_{i} * \gamma_{j}\right) \cup_{\mathrm{orb}} \gamma_{l}:=\left\langle\gamma_{i}, \gamma_{j}, \gamma_{l}\right\rangle_{0,3}^{X}
$$

or equivalently,

$$
\gamma_{i} * \gamma_{j}:=\sum_{l=1}^{N} \sum_{\beta}\left\langle\gamma_{i}, \gamma_{j}, \gamma_{l}\right\rangle_{0,3, \beta}^{X} P D\left(\gamma_{l}\right) q^{\omega(\beta)}
$$

where $P D(\cdot)$ denotes the Poincaré dual with respect to the pairing (3.1). Therefore, (3.2) with $n=3$ gives structure constants of this product. The associativity of $*$ is proved in [CR]. We remark that what we have defined is the small quantum cohomology of $X$ while the big quantum cohomology involves the full GromovWitten invariants.

\subsection{Elliptic orbifolds $\mathbb{P}_{a, b, c}^{1}$ and review on Satake-Takahashi's work}

We now focus on elliptic orbifolds with three cone points $\mathbb{P}_{a, b, c}^{1}$ and their GromovWitten potential. We have that $\mathbb{P}_{a, b, c}^{1}$ is elliptic if and only if $\frac{1}{a}+\frac{1}{b}+\frac{1}{c}=1$, and hence there are three elliptic orbifold projective lines $\mathbb{P}_{a, b, c}^{1}$ where $(a, b, c)$ are $(3,3,3),(2,3,6)$, and $(2,4,4)$. They are called elliptic since these orbifolds can be described as a global quotient of an elliptic curve $E$ by a finite cyclic group $G$.

We first fix the notation for generators of their orbifold cohomology rings in the following way. Let $w_{1}, w_{2}$, and $w_{3}$ be the three cone points $\mathbb{P}_{a, b, c}^{1}$ with isotropy groups $\mathbb{Z}_{a}, \mathbb{Z}_{b}$, and $\mathbb{Z}_{c}$, respectively. We fix a choice of a $\mathbb{Q}$-basis of $H_{\text {orb }}^{*}\left(\mathbb{P}_{a, b, c}^{1}, \mathbb{Q}\right)$, which is fairly standard. The $\mathbb{Q}$-basis

$$
\left\{1, \Delta_{1}^{1 / a}, \ldots, \Delta_{1}^{(a-1) / a}, \Delta_{2}^{1 / b}, \ldots, \Delta_{2}^{(b-1) / b}, \Delta_{3}^{1 / c}, \ldots, \Delta_{3}^{(c-1) / c},[\mathrm{pt}]\right\}
$$

of $H_{\text {orb }}^{*}\left(\mathbb{P}_{a, b, c}^{1}, \mathbb{Q}\right)$ is defined by the following conditions. The bases of a smooth sector are

$$
H_{\text {orb }}^{0}\left(\mathbb{P}_{a, b, c}^{1}, \mathbb{Q}\right)=\mathbb{Q}\langle 1\rangle, \quad H_{\text {orb }}^{2}\left(\mathbb{P}_{a, b, c}^{1}, \mathbb{Q}\right)=\mathbb{Q}\langle[\mathrm{pt}]\rangle
$$

For twist sectors, let $\Delta_{1}^{j / a} \in H_{\text {orb }}^{\frac{2 j}{a}}\left(\mathbb{P}_{a, b, c}^{1}, \mathbb{Q}\right), \Delta_{2}^{j / b} \in H_{\text {orb }}^{\frac{2 j}{b}}\left(\mathbb{P}_{a, b, c}^{1}, \mathbb{Q}\right)$, and $\Delta_{3}^{j / c} \in$ $H_{\text {orb }}^{\frac{2 j}{c}}\left(\mathbb{P}_{a, b, c}^{1}, \mathbb{Q}\right)$, which are supported at singular points $w_{1}, w_{2}$, and $w_{3}$, respectively. For $\boldsymbol{t}:=\sum t_{j, i} \Delta_{j}^{i}$, orbifold cup products with respect to these bases are given as follows:

$$
\int_{X}^{\mathrm{orb}} \Delta_{1}^{j / a} \cup \Delta_{1}^{k / a}=\frac{1}{a} \delta_{j+k-a, 0}, \quad \int_{X}^{\mathrm{orb}} \Delta_{1}^{j / b} \cup \Delta_{1}^{k / b}=\frac{1}{b} \delta_{j+k-b, 0},
$$




$$
\int_{X}^{\mathrm{orb}} \Delta_{1}^{j / c} \cup \Delta_{1}^{k / c}=\frac{1}{a} \delta_{j+k-c, 0}, \quad \int_{X}^{\mathrm{orb}} 1 \cup[\mathrm{pt}]=1,
$$

where $\delta_{i, j}$ is 1 if $i=j$ and 0 otherwise. The last cup product does not have any fraction since both 1 and [pt] live in a smooth (untwisted) section of $I X$.

\section{REMARK 3.8}

Readers are hereby warned that the Poincaré dual $P D\left(\Delta_{1}^{j / a}\right)$ of $\Delta_{1}^{j / a}$ is not $\Delta_{1}^{(a-j) / a}$, but $a \times \Delta_{1}^{(a-j) / a}$, and the same happens for $b$ and $c$. However, 1 and [pt] are still Poincaré dual to each other.

In the remaining part, we briefly recall the work of Satake and Takahashi [ST] on $\mathbb{P}_{3,3,3}^{1}$. We first give a description of $\mathbb{P}_{3,3,3}^{1}$ as a quotient of an elliptic curve. Let $E$ be the elliptic curve associated with the lattice $\mathbb{Z}\langle 1, \tau\rangle$ in $\mathbb{C}$ where $\tau=\exp \left(\frac{2 \pi \sqrt{-1}}{3}\right)$. Then the $\mathbb{Z}_{3}$-action on $\mathbb{C}$ generated by a rotation of $2 \pi / 3$ descends to $E$ since this action preserves the lattice $\mathbb{Z}\langle 1, \tau\rangle$. By taking quotients of $E$ via the induced $\mathbb{Z}_{3}$-action, we finally obtain the global quotient orbifold $\mathbb{P}_{3,3,3}^{1}=\left[E / \mathbb{Z}_{3}\right]$. (The shaded region in Figure $3($ a) represents a fundamental domain of the $\mathbb{Z}_{3}$-action on $E$.) Since each fixed point in $E$ has the isotropy group isomorphic to $\mathbb{Z}_{3}, \mathbb{P}_{3,3,3}^{1}$ has three cone points, each of which has $\mathbb{Z}_{3}$-singularity. We denote these singular points by $w_{1}, w_{2}$, and $w_{3}$, respectively. Therefore, the inertia orbifold $\mathcal{I} \mathbb{P}_{3,3,3}^{1}$ consists of the trivial sector together with three $B \mathbb{Z}_{3}$ 's (equivalent to $\left[\mathbb{Z}_{3} \ltimes\{*\}\right]$ ), which are associated with the $w_{i}$ 's.

\section{REMARK 3.9}

Consider the universal covering $\mathbb{C} \rightarrow E$ of the elliptic curve. The composition $p: \mathbb{C} \rightarrow E \rightarrow \mathbb{P}_{3,3,3}^{1}$ as well as the quotient map $E \rightarrow \mathbb{P}_{3,3,3}^{1}$ is a holomorphic orbifold covering map in the sense of [T] (see also Definition 2.5). Indeed, $p$ is the orbifold universal cover. We will use this fact crucially to classify holomorphic orbispheres in $\mathbb{P}_{3,3,3}^{1}$.

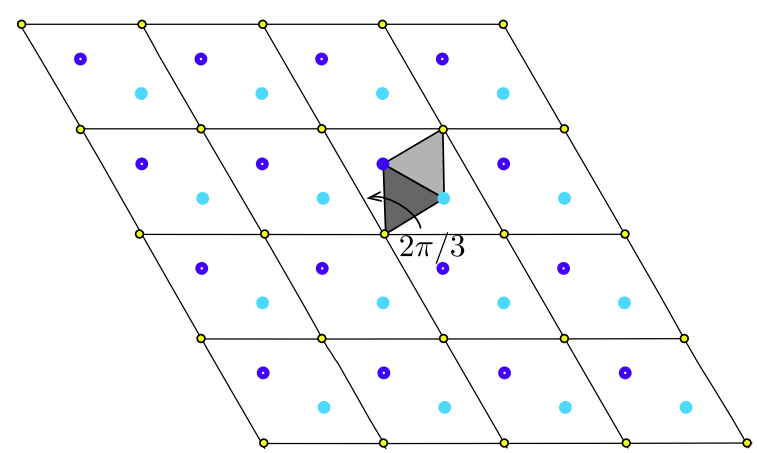

(a)

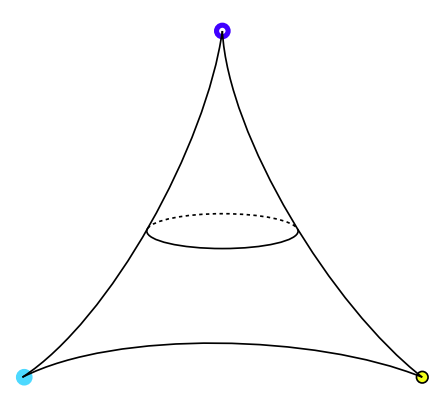

(b)

Figure 3. (a) The $\mathbb{Z}_{3}$-action on $E$ and (b) its quotient. 
Following the notation in $(3.4)$, the $\mathbb{Q}$-basis of $H_{\text {orb }}^{*}\left(\mathbb{P}_{3,3,3}^{1}, \mathbb{Q}\right)$ is given by $H_{\text {orb }}^{0}\left(\mathbb{P}_{3,3,3}^{1}, \mathbb{Q}\right)=\mathbb{Q}\langle 1\rangle, \quad H_{\text {orb }}^{2}\left(\mathbb{P}_{3,3,3}^{1}, \mathbb{Q}\right)=\mathbb{Q}\langle[\mathrm{pt}]\rangle$, $H_{\text {orb }}^{\frac{2}{3}}\left(\mathbb{P}_{3,3,3}^{1}, \mathbb{Q}\right)=\mathbb{Q}\left\langle\Delta_{1}^{1 / 3}, \Delta_{2}^{1 / 3}, \Delta_{3}^{1 / 3}\right\rangle, \quad H_{\text {orb }}^{\frac{4}{3}}\left(\mathbb{P}_{3,3,3}^{1}, \mathbb{Q}\right)=\mathbb{Q}\left\langle\Delta_{1}^{2 / 3}, \Delta_{2}^{2 / 3}, \Delta_{3}^{2 / 3}\right\rangle$, and the Poincaré pairing for $\Delta_{j}^{i}$ 's is determined by

$$
\int_{\mathbb{P}_{3,3,3}^{1}}^{\text {orb }} \Delta_{j}^{i} \cup_{\text {orb }} \Delta_{l}^{k}= \begin{cases}\frac{1}{3} & \text { if } i+k=1 \text { and } j=l, \\ 0 & \text { otherwise. }\end{cases}
$$

Satake and Takahashi [ST, Theorem 3.1] calculated the genus 0 GromovWitten potential of $\mathbb{P}_{3,3,3}^{1}$ and the quantum product term can be written as

$$
\begin{aligned}
& f_{0}(q):=\sum_{d \geq 0}\left\langle\Delta_{1}^{1 / 3}, \Delta_{2}^{1 / 3}, \Delta_{3}^{1 / 3}\right\rangle_{0,3, d} q^{d}=\frac{\eta\left(q^{9}\right)^{3}}{\eta\left(q^{3}\right)} \\
& f_{1}(q):=\sum_{d \geq 0}\left\langle\Delta_{1}^{1 / 3}, \Delta_{1}^{1 / 3}, \Delta_{1}^{1 / 3}\right\rangle_{0,3, d} q^{d}=\left(1+\frac{1}{3}\left(\frac{\eta(q)}{\eta\left(q^{9}\right)}\right)^{3}\right) f_{0}(q) .
\end{aligned}
$$

Here, $\eta(q)$ is Dedekind's eta function

$$
\eta(\tau):=\exp \left(\frac{\pi \sqrt{-1} \tau}{2}\right) \prod_{n=1}^{\infty}\left(1-q^{n}\right), \quad q=\exp (2 \pi \sqrt{-1} \tau),
$$

for $\tau \in \mathbb{H}:=\{\tau \in \mathbb{C} \mid \operatorname{Im}(\tau)>0\}$.

Write $f_{0}(q)=\sum_{N \geq 1} a_{N} q^{N}$. The Fourier coefficients $a_{N}$ of $f_{0}$ depend on the prime factorization of $N$ (or more precisely the quadratic reciprocity of $N$ ) and are given by

$$
a_{N}= \begin{cases}0 & \text { if } n>0 \\ 0 & \text { if one of } m_{j} \text { is odd } \\ \left(n_{1}+1\right) \cdots\left(n_{k}+1\right) & \text { otherwise }\end{cases}
$$

for $N=3^{n} p_{1}^{n_{1}} \cdots p_{k}^{n_{k}} q_{1}^{m_{1}} \cdots q_{l}^{m_{l}}$ where $p_{i}$ is a prime number with $p_{i} \equiv 1(\bmod 3)$, and $q_{i}$ is a prime number with $q_{i} \equiv 2(\bmod 3)($ see $[\mathrm{S}])$. The Fourier coefficients of $f_{1}$ also have a similar description which we will give in Appendix A.

We also provide the first few terms of $f_{0}$ and $f_{1}$ to give readers an impression:

$$
\begin{aligned}
& f_{0}=q+q^{4}+2 q^{7}+2 q^{13}+q^{16}+2 q^{19}+O\left(q^{24}\right), \\
& f_{1}=\frac{1}{3}+2 q^{3}+2 q^{9}+2 q^{12}+4 q^{21}+O\left(q^{24}\right) .
\end{aligned}
$$

\section{Holomorphic orbifold maps}

As mentioned in the introduction, our main goal is to compute the (quantum) product structure of $H_{\text {orb }}^{*}\left(\mathbb{P}_{a, b, c}^{1}, \mathbb{Q}\right)$ where $(a, b, c)$ is either $(3,3,3),(2,3,6)$, or $(2,4,4)$. Throughout the section, $\mathbb{P}_{a, b, c}^{1}$ denotes one of the elliptic orbifolds $\mathbb{P}_{3,3,3}^{1}$, $\mathbb{P}_{2,3,6}^{1}$, or $\mathbb{P}_{2,4,4}^{1}$. In order to do this, we have to count holomorphic orbispheres in (or stable maps into) $\mathbb{P}_{a, b, c}^{1}$ with three markings. In this section, we first characterize these holomorphic maps and find their properties which are useful 
for classifying holomorphic orbispheres in $\mathbb{P}_{a, b, c}^{1}$. We will see that if $\left(f,\left(\mathbb{P}^{1}, \boldsymbol{z}\right), \xi\right)$ is a nonconstant orbifold stable map into elliptic $\mathbb{P}_{a, b, c}^{1}$ of type $\boldsymbol{x}$, then $\boldsymbol{z}$ cannot contain a smooth point. Thus, we may assume that $\boldsymbol{x}$ is a triple of twisted sectors (see the discussion after the proof of Lemma 4.1 below).

Recall that the type $\boldsymbol{x}$ determines the virtual dimension of a component of the moduli of orbifold stable maps containing $\left(f,\left(\mathbb{P}^{1}, \boldsymbol{z}\right), \xi\right)$ as well as the orbifold structure of the domain orbi-Riemann sphere $\left(\mathbb{P}^{1}, \boldsymbol{z}\right)$ (see Remark 3.7). In fact, the virtual dimension is given as

$$
\operatorname{vir} . \operatorname{dim} \overline{\mathcal{M}}_{0,3}\left(\mathbb{P}_{a, b, c}^{1}, J, \beta, \boldsymbol{x}\right)=2-2 \iota(\boldsymbol{x}) .
$$

As we only consider the 0 -dimensional moduli for the quantum product, this gives a restriction on the type, that is, $\iota(\boldsymbol{x})=1$. If we impose an additional condition on the degrees of inputs for a holomorphic orbisphere of type $\boldsymbol{x}$ with $\iota(\boldsymbol{x})=1$, then we can show that it is actually an orbifold covering. This will be shown in Section 4.2. As the first step, we show that there is no contribution to the quantum product from a degenerate orbisphere, which is an element lying on the boundary of $\overline{\mathcal{M}}_{0,3, \beta}\left(\mathbb{P}_{a, b, c}^{1}\right)$.

\subsection{Considerations on degenerate maps}

Note that the $\Delta_{\circ}^{i}$ 's are cohomology classes of nontrivial sectors of $\mathbb{P}_{a, b, c}^{1}$. We want to show that all the holomorphic orbispheres $u:\left(\Sigma, \boldsymbol{z}=\left(z_{1}, z_{2}, z_{3}\right)\right) \rightarrow \mathbb{P}_{a, b, c}^{1}$ of appropriate type $\boldsymbol{x}=\left(x_{1}, x_{2}, x_{3}\right)$, a triple of twisted sectors of $\mathbb{P}_{a, b, c}^{1}$, cannot have any nodal singularity. More precisely, the above "appropriate" means that the $\boldsymbol{x}$ is a type with vir.dim $\overline{\mathcal{M}}_{0,3}\left(\mathbb{P}_{a, b, c}^{1}, J, \beta, \boldsymbol{x}\right)=0$ for all $\beta$. Here, since $\mathbb{P}_{a, b, c}^{1}$ is elliptic, the virtual dimension does not depend on $\beta$ (Remark 3.7).

\section{LEMMA 4.1}

There are no degenerate (i.e., nodal) holomorphic orbispheres which are nonconstant and contribute to $\left\langle\Delta_{\circ}^{i}, \Delta_{\bullet}^{j}, \Delta_{\diamond}^{k}\right\rangle_{0,3}^{\mathbb{P}_{a, b, c}^{1}}$ for $i+j+k=1$.

Proof

There are two classes of degenerate maps which are possibly contained in the boundary of the moduli space $\overline{\mathcal{M}}_{0,3}\left(\mathbb{P}_{a, b, c}^{1}, J, \beta, \boldsymbol{x}\right)$ :

(1) $u_{1}: \mathbb{P}_{\alpha, \beta, \bullet}^{1} \sqcup_{\bullet} \mathbb{P}_{\bullet}^{1}, \delta \mathbb{P}_{a, b, c}^{1}$,

(2) $u_{2}: \mathbb{P}_{\alpha, \beta, \delta, \circ}^{1} \sqcup_{\circ} \mathbb{P}_{\circ}^{1} \rightarrow \mathbb{P}_{a, b, c}^{1}$,

where $\bullet$ and $\circ$ are the order of the local isotropy group of the nodal point (see Figure 4). Note that $u_{i}(i=1,2)$ is a nonconstant map when restricted to the second component of the domain, since $u_{i}$ should be stable. We claim that there cannot exist such maps into $\mathbb{P}_{a, b, c}^{1}$.

First, consider the case of $u: \mathbb{P}_{\delta, \delta}^{1} \rightarrow \mathbb{P}_{a, b, c}^{1}(\bullet=\delta$ in (1)). Since the quotient map $\pi: \mathbb{P}^{1} \rightarrow \mathbb{P}_{\delta, \delta}^{1}$ (by the obvious $\mathbb{Z}_{\delta}$-action on $\mathbb{P}^{1}$ ) is a holomorphic orbifold covering and $\pi_{1}\left(\mathbb{P}^{1}\right)=0$, there is a holomorphic map $\tilde{u}$ which makes the following 


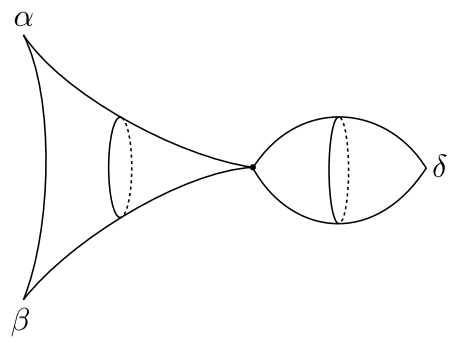

(a)

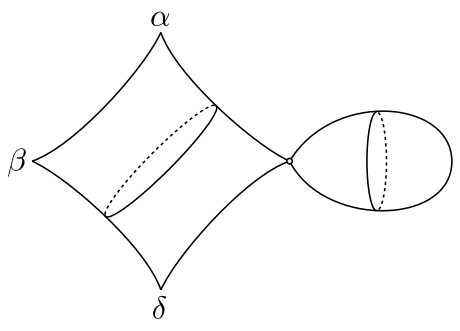

(b)

Figure 4. Domains of degenerate maps: (a) $\mathbb{P}_{\alpha, \beta, \bullet}^{1} \sqcup_{\bullet} \mathbb{P}_{\bullet, \delta}^{1}$ and (b) $\mathbb{P}_{\alpha, \beta, \delta, \circ}^{1} \sqcup_{\circ} \mathbb{P}_{\circ}^{1}$.

diagram commute (using Proposition 2.8 and Lemma 2.10).

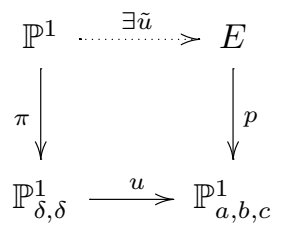

Note that the image of $\tilde{u}$ must be homotopic to a constant map since $\pi_{2}(E)=0$, and hence $\tilde{u}$ is a constant map from the holomorphicity. This contradicts the stability of the map $u$, and hence there is no such holomorphic map $u$. A similar argument shows that $u: \mathbb{P}^{1} \rightarrow \mathbb{P}_{a, b, c}^{1}(\circ=1$ in $(2))$ cannot exist.

The remaining case is when the second component of the domain is not a good orbifold, namely, $u: \mathbb{P}_{m p, m q}^{1} \rightarrow \mathbb{P}_{a, b, c}^{1}$ for some natural numbers $p, q$, and $m$ satisfying $\operatorname{gcd}(p, q)=1$ and $p q \neq 1$. Consider the holomorphic quotient map $\pi: \mathbb{P}_{p, q}^{1} \rightarrow \mathbb{P}_{m p, m q}^{1}$, and consider the holomorphic map $v:=u \circ \pi$.

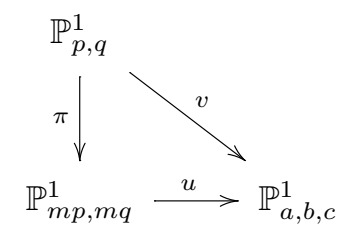

Let $x \in \mathbb{P}_{m p, m q}^{1}$ be an orbisingular point, and let $\tilde{x} \in \mathbb{P}_{p, q}^{1}$ be the element in $\pi^{-1}(x)$. We may assume that $\tilde{x}$ and $u(x)$ have isotropy groups $\mathbb{Z}_{p}$ for some $p \neq 1$ and $\mathbb{Z}_{a}$, respectively. Then from the definition of an orbifold map, the map $v$ should be lifted locally to an equivariant map $\widetilde{v}$ on the local uniformizing charts

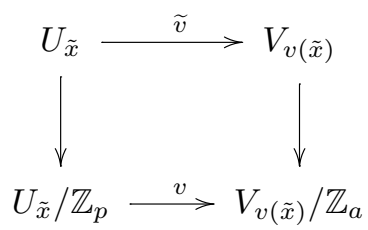


and the induced group homomorphism between isotropy groups $\phi_{v}: \mathbb{Z}_{p} \rightarrow \mathbb{Z}_{a}$ should be injective since $\phi_{v}=\phi_{u} \circ \phi_{\pi}: \mathbb{Z}_{p} \stackrel{\times m}{\rightarrow} \mathbb{Z}_{m p} \rightarrow \mathbb{Z}_{a}$ is a composition of two injective morphisms. (The injectivity of the second map comes from the definition of an orbifold map. See Definition 2.2.) Hence, the generator $g$ of $\pi_{1}^{\text {orb }}\left(U_{\tilde{x}} / \mathbb{Z}_{p}\right)$ should be mapped to an order $p$ element of $\pi_{1}^{\text {orb }}\left(V_{v(\tilde{x})} / \mathbb{Z}_{a}\right)$. However, from van Kampen's theorem, $\pi_{1}^{\text {orb }}\left(\mathbb{P}_{p, q}^{1}\right)=\{0\}$, so the image of $g$ in $\pi_{1}^{\text {orb }}\left(\mathbb{P}_{p, q}^{1}\right)$ is zero whereas $\pi_{1}^{\text {orb }}\left(\mathbb{P}_{a, b, c}^{1}\right)=\left\langle g_{1}, g_{2}, g_{3} \mid g_{1}^{a}=g_{2}^{b}=g_{3}^{c}=g_{1} g_{2} g_{3}=1\right\rangle$ is nontrivial. Note that the homomorphism $\pi_{1}^{\text {orb }}\left(V_{v(\tilde{x})} / \mathbb{Z}_{a}\right) \rightarrow \pi_{1}^{\text {orb }}\left(\mathbb{P}_{a, b, c}^{1}\right)$ induced from the inclusion map $\iota: V_{v(\tilde{x})} \rightarrow \mathbb{P}_{a, b, c}^{1}$ is injective, since $\mathbb{P}_{a, b, c}^{1}$ is a good orbifold (see, e.g., [D, Proposition 1.18]). This gives a contradiction.

Consider an orbifold stable map $\left(f,\left(\mathbb{P}^{1}, \boldsymbol{z}\right), \xi\right)$ with three markings of type $\boldsymbol{x}$. If there is a smooth point in $\boldsymbol{z}$, then $f$ can be thought of as a map from an orbisphere with two singular points. Then exactly the same argument in the proof of Lemma 4.1 implies that $f$ is indeed a constant map.

\subsection{Orbifold coverings of $\mathbb{P}_{a, b, c}^{1}$ contributing to the quantum product}

In this section, we prove that holomorphic orbispheres satisfying certain properties become orbifold covering maps. Most of the holomorphic orbispheres contributing to the quantum product of elliptic $\mathbb{P}_{a, b, c}^{1}$ will satisfy these properties, as will be shown later. (There is only one exceptional case for $\mathbb{P}_{2,3,6}^{1}$ where nontrivial holomorphic orbispheres from the hyperbolic orbifold $\mathbb{P}_{3,6,6}^{1}$ contribute to the quantum product of $\mathbb{P}_{2,3,6}^{1}$.)

Let $u$ be a holomorphic orbisphere from $\mathbb{P}_{\alpha, \beta, \delta}^{1}$ to $\mathbb{P}_{a, b, c}^{1}$, consider the universal covering map $\pi: \widetilde{\mathbb{P}_{\alpha, \beta, \delta}^{1}} \rightarrow \mathbb{P}_{\alpha, \beta, \delta}^{1}$, and consider $p: \mathbb{C} \rightarrow \mathbb{P}_{a, b, c}^{1}$. Here, $p$ is a holomorphic map since the complex structure on $\mathbb{P}_{a, b, c}^{1}$ comes from that on its universal cover. From orbifold covering theory, we obtain a lifting $\tilde{u}$ of $u \circ \pi$ of the underlying orbifold morphism $u: \mathbb{P}_{\alpha, \beta, \delta}^{1} \rightarrow \mathbb{P}_{a, b, c}^{1}$ :

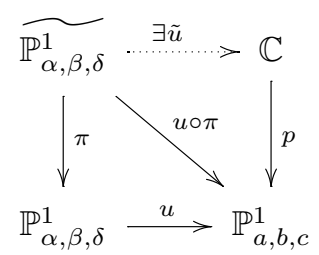

For each equivalence class $[u] \in \mathcal{M}_{0,3, \beta}^{\text {reg }}\left(\mathbb{P}_{a, b, c}^{1}\right)$, if we choose a representative $u$ of $[u]$ by fixing the location of three special points on the domain (denoted by $\mathbb{P}_{\alpha, \beta, \delta}^{1}$ ), then there is no further equivalence relation since there is a unique automorphism which sends the given three points to the others. For such $u$, the lifting $\tilde{u}$ is holomorphic, since it is locally holomorphic.

To avoid notational complexity, let us write $X$ for $\mathbb{P}_{a, b, c}^{1}$, and consider a triple of twisted sectors $\boldsymbol{x}=\left(X_{\left(g_{1}\right)}, X_{\left(g_{2}\right)}, X_{\left(g_{3}\right)}\right)$. Let $G_{i}$ be an isotropy group of a point in $X_{\left(g_{i}\right)}$ which is defined up to conjugacy. Since $X$ is 1-dimensional, the age of an 
element of $G_{i}$ is given by $\iota_{\left(g_{i}\right)}=\frac{l_{i}}{\left|G_{i}\right|}$ for some $l_{i} \in\left\{1, \ldots,\left|G_{i}\right|-1\right\}$. Since we only count 0 -dimensional strata of moduli of orbifold stable maps for the quantum product, we assume that

$$
\sum_{i=1}^{3} \iota\left(g_{i}\right)=1 .
$$

From Definition 2.5, we see that the necessary condition for $u$ to be an orbifold covering map is that

$$
\iota_{\left(g_{i}\right)}^{-1} \in \mathbb{Z}, \quad i=1,2,3,
$$

or equivalently $l_{i}|| G_{i} \mid$ for $i=1,2,3$. Nonetheless, this condition (4.5) is indeed sufficient to guarantee that $u$ is an orbifold covering map.

\section{LEMMA 4.2}

If $u$ is a nonconstant holomorphic orbifold stable map from $\left(\mathbb{P}^{1}, \boldsymbol{z}, \boldsymbol{m}\right)$ to $X$ of the type $\boldsymbol{x}$ satisfying (4.4) and (4.5), then $u$ is an orbifold covering map. Here, $\boldsymbol{z}=\left(z_{1}, z_{2}, z_{3}\right)$ is a triple of marked points, and $\boldsymbol{m}=\left(m_{1}, m_{2}, m_{3}\right)$ is the triple of orders of isotropy groups of $\boldsymbol{z}$.

\section{Proof}

Recall that any nonconstant holomorphic map between Riemann surfaces is a branched covering. We want to show that this map is an orbifold covering (see Definition 2.5).

Consider three orbisingular points $\left\{w_{1}, w_{2}, w_{3}\right\}$ in the target space, and consider their inverse image $u^{-1}\left(w_{i}\right)$. Since $\boldsymbol{x}$ consists of twisted sectors, there is a function $I:\{1,2,3\} \rightarrow\{1,2,3\}$ such that $u\left(z_{i}\right)=w_{I(i)} \in X_{\left(g_{i}\right)}$. We denote the number of points in $u^{-1}\left(w_{i}\right)-\left\{z_{1}, z_{2}, z_{3}\right\}$ by $m\left(w_{i}\right)$.

Let $U_{i}$ be an open neighborhood of $z_{i}$ with uniformizing system $\left(\widetilde{U}_{i}, \mathbb{Z}_{m_{i}}, b r_{i}\right)$, where $b r_{i}: z \mapsto z^{m_{i}}$, and let $V$ be an open neighborhood of $w_{I(i)}$ uniformized by $\left(\widetilde{V}, \mathbb{Z}_{\left|G_{i}\right|}, b r\right)$ for $b r: z \mapsto z^{\left|G_{i}\right|}$. By the definition of orbifold morphisms, there is a local holomorphic lifting $\tilde{u}$ of $u$ such that the diagram

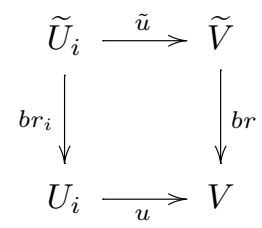

commutes. Then, from (4.5) and the injectivity of $\mathbb{Z}_{m_{i}} \rightarrow \mathbb{Z}_{\left|G_{j}\right|}$ which maps 1 to $l_{i}$, one can check that $m_{i} l_{i}=\left|G_{i}\right|$ and $\tilde{u}(z)=z^{m_{i} a_{i}+1}$ for some $a_{i} \in \mathbb{N}_{\geq 0}$. Then $u(w)=w^{\left|G_{i}\right| a_{i}+l_{i}}$ where $w$ is a local holomorphic coordinate $w=z^{m_{i}}$ of the underlying space of $\mathbb{P}_{a, b, c}^{1}$.

Since any orbifold Riemann surface $\left(\Sigma_{g}, \boldsymbol{z}\right)$ is analytically isomorphic to a smooth Riemann surface $\Sigma_{g}$, the orbifold map $u$ can be regarded as a branched covering between underlying spaces $\mathbb{C P}^{1}$. Hence, the ramification index at $z_{i}$ is 
$\left|G_{i}\right| a_{i}+l_{i}$ for $i=1,2,3$. In the inverse image $u^{-1}\left(w_{1}\right)$, every point except the orbisingular points $z_{i}$ have ramification indices that are multiples of $a$, say, $a e_{j}$ for some $e_{j} \in \mathbb{N}$ and $j=1, \ldots, m\left(w_{1}\right)$. Similarly, in $u^{-1}\left(w_{2}\right)$ and $u^{-1}\left(w_{3}\right)$, there are $m\left(w_{2}\right)$ and $m\left(w_{3}\right)$ points with ramification indices $b f_{k}$ and $c g_{l}$ for $1 \leq k \leq m\left(w_{2}\right)$ and $1 \leq l \leq m\left(w_{3}\right)$, respectively.

We apply the Riemann-Hurwitz formula to $u$ to obtain

$$
\begin{aligned}
2 \leq & 2 d-\left\{\sum_{i=1}^{3}\left(\left|G_{i}\right| a_{i}+l_{i}-1\right)+\sum_{j=1}^{m\left(w_{1}\right)}\left(a e_{j}-1\right)\right. \\
& \left.+\sum_{k=1}^{m\left(w_{2}\right)}\left(b f_{k}-1\right)+\sum_{l=1}^{m\left(w_{3}\right)}\left(c g_{l}-1\right)\right\},
\end{aligned}
$$

where $d$ is the degree of $u$. (Here, the 2 on the left-hand side is the topological Euler characteristic of $\mathbb{P}_{a, b, c}^{1}$. $)$ If $u$ does not have any branching outside $u^{-1}\left(w_{1}\right) \cup$ $u^{-1}\left(w_{2}\right) \cup u^{-1}\left(w_{3}\right)$, then the equality holds in (4.6). Since $d$ is the weighted count of the number of points in the fiber $u^{-1}\left(w_{i}\right)$ of $u$, we have

$$
\begin{aligned}
d & =\sum_{i \in I^{-1}(1)}\left(\left|G_{i}\right| a_{i}+l_{i}\right)+a \sum_{j=1}^{m\left(w_{1}\right)} e_{j} \\
& =\sum_{i \in I^{-1}(2)}\left(\left|G_{i}\right| a_{i}+l_{i}\right)+b \sum_{k=1}^{m\left(w_{2}\right)} f_{k} \\
& =\sum_{i \in I^{-1}(3)}\left(\left|G_{i}\right| a_{i}+l_{i}\right)+c \sum_{l=1}^{m\left(w_{3}\right)} g_{l},
\end{aligned}
$$

and hence, by inserting (4.7) in (4.6),

$$
d \leq 1+\sum_{j=1}^{3} m\left(w_{j}\right) .
$$

Then, (4.7) together with $e_{j} \geq 1$ implies that

$$
d \geq \sum_{i \in I^{-1}(1)}\left(\left|G_{i}\right| a_{i}+l_{i}\right)+a m\left(w_{1}\right),
$$

and similar inequalities also hold for $w_{2}$ and $w_{3}$. Combining (4.8) and (4.9), we have

$$
\begin{aligned}
& a b c\left(\sum_{i=1}^{3} m\left(w_{i}\right)\right)+b c \sum_{i \in I^{-1}(1)}\left(\left|G_{i}\right| a_{i}+l_{i}\right) \\
& \quad+c a \sum_{i \in I^{-1}(2)}\left(\left|G_{i}\right| a_{i}+l_{i}\right)+a b \sum_{i \in I^{-1}(3)}\left(\left|G_{i}\right| a_{i}+l_{i}\right) \\
& \leq(b c+c a+a b) d=a b c d,
\end{aligned}
$$

where the last equality follows from $\frac{1}{a}+\frac{1}{b}+\frac{1}{c}=1$. 
Note that $\sum_{i=1}^{3} \frac{l_{i}}{\left|G_{i}\right|}=1$ from the condition (4.4). Hence

$$
b c \sum_{i \in I^{-1}(1)} l_{i}+c a \sum_{i \in I^{-1}(2)} l_{i}+a b \sum_{i \in I^{-1}(3)} l_{i}=a b c .
$$

Since $\left|G_{i}\right|=a$ if $i \in I^{-1}(1)$, we have (along with similar equalities for the other two cases)

$$
b c \sum_{i \in I^{-1}(1)}\left|G_{i}\right| a_{i}+c a \sum_{i \in I^{-1}(2)}\left|G_{i}\right| a_{i}+a b \sum_{i \in I^{-1}(3)}\left|G_{i}\right| a_{i}=a b c \sum_{i=1}^{3} a_{i} .
$$

Combining (4.10) with (4.8), (4.11), and (4.12), we have that

$$
a b c\left(\sum_{i=1}^{3} m\left(w_{i}\right)+\sum_{i=1}^{3} a_{i}+1\right) \leq a b c\left(1+\sum_{j=1}^{3} m\left(w_{j}\right)\right) .
$$

Hence, $a_{1}=a_{2}=a_{3}=0$ from the nonnegativity of the $a_{i}$ 's.

If we do not use inequality (4.9) and proceed, then we have the more precise estimate

$$
a b c\left(\sum_{j=1}^{m\left(w_{1}\right)} e_{j}+\sum_{k=1}^{m\left(w_{2}\right)} f_{k}+\sum_{l=1}^{m\left(w_{3}\right)} g_{l}+\sum_{i=1}^{3} a_{i}+1\right) \leq a b c\left(1+\sum_{j=1}^{3} m\left(w_{j}\right)\right),
$$

which implies $e_{j}=f_{k}=g_{l}=1$ for all $j, k, l$. Therefore, $u$ is an orbifold covering.

\section{REMARK 4.3}

For the case of $c_{1}(T X)<0$ (i.e., $X=\mathbb{P}_{a, b, c}^{1}$ is hyperbolic), the same lemma also holds since (4.10) is still valid. However, if $c_{1}(T X)>0$ (i.e., $X$ is spherical), then the argument in Lemma 4.2 is no longer true.

\subsection{Regularity of holomorphic maps}

Finally, we show that holomorphic orbispheres which become orbifold coverings of elliptic $\mathbb{P}_{a, b, c}^{1}$ are Fredholm regular. We first recall the definition of the desingularization of an orbibundle, and then we examine some properties of the desingularized bundle which will be used for proving the regularity.

Let $(\Sigma, \boldsymbol{z}, \boldsymbol{m})$ be an orbi-Riemann surface, and consider an orbibundle $E \rightarrow$ $(\Sigma, \boldsymbol{z}, \boldsymbol{m})$. The desingularization of $E$ is defined as follows. For each disc neighborhood $D_{i}$ of orbisingular points $z_{i}$ in $\boldsymbol{z}, E$ can be uniformized by $\left(D_{i} \times \mathbb{C}^{n}, \mathbb{Z}_{m_{i}}, \pi\right)$ so that the action is linear and diagonal. Hence, the action can be written as

$$
e^{\frac{2 \pi \sqrt{-1}}{m_{i}}} \cdot(z, f)=\left(e^{\frac{2 \pi \sqrt{-1}}{m_{i}}} z, \operatorname{diag}\left(e^{\frac{2 \pi \sqrt{-1} m_{i, 1}}{m_{i}}}, \ldots, e^{\frac{2 \pi \sqrt{-1} m_{i, n}}{m_{i}}}\right) f\right)
$$

for some integers $0 \leq m_{i, j}<m_{i}(j=1, \ldots, n)$. Let $\Phi_{i}: D^{*} \times \mathbb{C}^{n} \rightarrow D^{*} \times \mathbb{C}^{n}$ be a $\mathbb{Z}_{m_{i}}$-equivariant map over the punctured disc $D^{*}=D_{i}-\{0\}$ defined by

$$
\Phi_{i}\left(z, f_{1}, \ldots, f_{n}\right)=\left(z^{m_{i}}, z^{-m_{i, 1}} f_{1}, \ldots, z^{-m_{i, n}} f_{n}\right),
$$

where $\mathbb{Z}_{m_{i}}$ trivially acts on the right-hand side. Consider the natural map $\phi$ : $(\Sigma, \boldsymbol{z}, \boldsymbol{m}) \rightarrow|\Sigma|$ which can be written as $z \rightarrow z^{m_{i}}$ over each $D_{i}$. Then the local 
holomorphic chart on $|\Sigma|$ is $w=z^{m_{i}}$ over each $\phi\left(D_{i}\right)$. We construct a complex vector bundle $|E|$ over the underlying space $|\Sigma|$ of $(\Sigma, \boldsymbol{z}, \boldsymbol{m})$ by extending the complex vector bundle over $|\Sigma|-\left\{z_{1}, \ldots, z_{k}\right\}$ whose trivialization is given by the right-hand side of (4.15).

Chen and Ruan [CR1] observed that the first Chern number of an orbibundle is the sum of the first Chern number of its desingularization and the ages of representations which are induced from local trivialization of the orbibundle over orbisingular points. More precisely, let $E$ be an orbibundle over a closed orbiRiemann surface $(\Sigma, \boldsymbol{z}, \boldsymbol{m})$, and set $\boldsymbol{m}=\left(m_{1}, \ldots, m_{k}\right)$ for $m_{i} \in \mathbb{Z}_{\geq 0}$. Then for each orbisingular point $z_{i}$, the induced representation $\rho_{i}: \mathbb{Z}_{m_{i}} \rightarrow \operatorname{End}\left(\mathbb{C}^{n}\right)$ can be written as

$$
\rho_{i}\left(e^{\frac{2 \pi \sqrt{-1}}{m_{i}}}\right)=\operatorname{diag}\left(e^{\frac{2 \pi \sqrt{-1} m_{i, 1}}{m_{i}}}, \ldots, e^{\frac{2 \pi \sqrt{-1} m_{i, n}}{m_{i}}}\right)
$$

for some integers $0 \leq m_{i, j}<m_{i}(j=1, \ldots, n)$. Then

$$
c_{1}(E)([\Sigma])=c_{1}(|E|)([\Sigma])+\sum_{i=1}^{k} \sum_{j=1}^{n} \frac{m_{i, j}}{m_{i}} .
$$

For a holomorphic orbibundle $E \rightarrow X$, let us denote sheaves of holomorphic sections of $E$ and $|E|$ over $X$ and $|X|$ by $\mathcal{O}(E)$ and $\mathcal{O}(|E|)$, respectively. Then we have $\mathcal{O}(E)=\mathcal{O}(|E|)$ (see [CR1, Proposition 4.2.2]) from the removability of isolated singularities of $J$-holomorphic maps. In detail, if $g: D_{i} \rightarrow \mathbb{C}^{n}$ is a local holomorphic section over $|E|_{D_{i}} \rightarrow D_{i}$, then $g(w)=\left(g_{1}(w), \ldots, g_{n}(w)\right)$ for some holomorphic maps $g_{i}: D_{i} \rightarrow \mathbb{C}$ with respect to the trivialization taken as above. If we pull back this section via $\Phi_{i}$, then the corresponding section on $\left.E\right|_{D_{i}} \rightarrow D_{i}$ is the holomorphic map $f: D_{i} \rightarrow \mathbb{C}^{n}$ whose components are $f_{j}(z)=z^{m_{i, j}} g_{j}\left(z^{m_{i}}\right)$ for each $j=1, \ldots, n$.

Conversely, let $f=\left(f_{1}, \ldots, f_{n}\right): D_{i} \rightarrow \mathbb{C}^{n}$ be a given local holomorphic section on the orbibundle $\left.E\right|_{D_{i}} \rightarrow D_{i}$, that is, $f$ is a $\mathbb{Z}_{m_{i}}$-equivariant holomorphic section. Define a map $g: D^{*} \rightarrow \mathbb{C}^{n}$ whose components are $g_{j}(w):=z^{-m_{i, j}} f_{j}(z)$ for $z=w^{\frac{1}{m_{i}}}$. Note that the $\mathbb{Z}_{m_{i}}$-equivariantness of $f$ says that the section $g$ is well defined, although there is an ambiguity on the choice of branch cut for $z=w^{\frac{1}{m_{i}}}$. Moreover, if we expand the holomorphic function $f_{j}$ as

$$
f_{j}(z)=\sum_{n=0}^{\infty} a_{n, j} z^{n}
$$

then $a_{n, j}=0$ unless $n \equiv m_{i, j}$ modulo $m_{i}$. Thus, $\left|\frac{f_{j}(z)}{z^{m} i, j}\right|$ is bounded on $D_{i}$, and we conclude that $g$ can be extended to a holomorphic section over $D_{i}$ using the Riemann extension theorem. One can easily check that this process is the inverse of the pullback via $\Phi_{i}$, which gives $\mathcal{O}(E)=\mathcal{O}(|E|)$.

Now we prove the Fredholm regularity of holomorphic orbispheres in elliptic $\mathbb{P}_{a, b, c}^{1}$, which are orbifold coverings. This will imply that the relevant moduli space of holomorphic orbispheres is smooth, and hence justify our direct counting of holomorphic orbispheres, later. 


\section{PROPOSITION 4.4}

Let $X$ be an elliptic orbisphere, and let $(\Sigma, \boldsymbol{z})$ be a domain Riemann orbicurve. If $u: \Sigma \rightarrow X$ is a holomorphic orbisphere satisfying (4.4) and (4.5), then $u$ is regular.

\section{Proof}

Consider the pullback orbibundle $u^{*} T X \rightarrow \Sigma$, and consider the linearized $\bar{\partial}$ operator $D \bar{\partial}_{J}(u): C^{\infty}\left(u^{*} T X\right) \rightarrow \Omega^{0,1}\left(u^{*} T X\right)$. Since $J$ is integrable, $D \bar{\partial}_{J}(u)=\bar{\partial}_{J}$. Hence, it is sufficient to show that $H_{\bar{\partial}}^{0,1}\left(X, u^{*} T X\right)=0$.

Note that the first Chern number of the tangent bundle of $X$ is zero, and for any orbifold covering $u$, that of $u^{*} T X$ is also zero. From this, we can see that the desingularized bundle of $u^{*} T X,\left|u^{*} T X\right|$, has (desingularized) Chern number -1 since the second term on the right-hand side of (4.16) is 1 from (4.4). That is, $c_{1}\left(\left|u^{*} T X\right|\right)=-1$. Since $X$ is a complex orbifold and $u$ is a holomorphic orbimap, $u^{*} T X$ is a holomorphic orbibundle over $\Sigma$. The desingularization of a holomorphic orbibundle is also holomorphic. From [McS, Lemma 3.5.1], this implies that the holomorphic line bundle $\left|u^{*} T X\right|$ has vanishing cohomology group $H_{\bar{\partial}}^{0,1}\left(|\Sigma|,\left|u^{*} T X\right|\right)$. As the sheaf of holomorphic sections of $\left|u^{*} T X\right|$ is the same as the sheaf of (orbifold) holomorphic sections of $u^{*} T X$ on $\Sigma$, we have the vanishing of $H_{\bar{\partial}}^{0,1}\left(\Sigma, u^{*} T X\right)$. More precisely,

$$
\begin{aligned}
H_{\bar{\partial}}^{0,1}\left(|\Sigma|,\left|u^{*} T X\right|\right) & \cong H^{1}\left(|\Sigma|, \mathcal{O}\left(\left|u^{*} T X\right|\right)\right) \\
& \cong H^{1}\left(\Sigma, \mathcal{O}\left(u^{*} T X\right)\right) \\
& \cong H_{\bar{\partial}}^{0,1}\left(\Sigma, u^{*} T X\right)
\end{aligned}
$$

For the last isomorphism, note that the two-term complex $\bar{\partial}_{J}: C^{\infty}\left(u^{*} T X\right) \rightarrow$ $\Omega^{0,1}\left(u^{*} T X\right)$ is a fine resolution for the sheaf of holomorphic sections $\mathcal{O}\left(u^{*} T X\right)$ as in the smooth case.

\section{REMARK 4.5}

Even if $u$ is not an orbifold covering, we still have $c_{1}\left(u^{*} T X\right) \geq c_{1}(T X)$. (Indeed, we can improve this inequality by considering the degree of $u$.) Therefore, the above proposition also holds as long as $X$ has a nonnegative first Chern number.

For example, when we calculate the quantum cohomology of $\mathbb{P}_{2,3,6}^{1}$, we need to count holomorphic orbispheres $u: \mathbb{P}_{3,6,6}^{1} \rightarrow \mathbb{P}_{2,3,6}^{1}$ which are not orbifold covering maps. These orbispheres are also Fredholm regular by exactly the same argument.

\section{The quantum cohomology ring of $\mathbb{P}_{3,3,3}^{1}$}

In this section, we explicitly compute the product structure on $Q H_{\text {orb }}^{*}\left(\mathbb{P}_{3,3,3}^{1}, \mathbb{Q}\right)$, which proves Theorem 1.1. For this, we first classify holomorphic orbispheres in $\mathbb{P}_{3,3,3}^{1}$ (Section 5.1). Recall from Lemma 4.1 and Proposition 4.4 that these stable maps, in fact, are maps from a single orbisphere component and are regular. Thus, by counting holomorphic orbispheres inside $\mathbb{P}_{3,3,3}^{1}$, we obtain the 3-point 
Gromov-Witten invariant for $\mathbb{P}_{3,3,3}^{1}$ which combined with the constant map contributions (Section 5.4) gives rise to the quantum product on $Q H_{\text {orb }}^{*}\left(\mathbb{P}_{3,3,3}^{1}, \mathbb{Q}\right.$ ). One interesting feature is that one can relate these orbispheres with the solutions of a certain Diophantine equation. In Section 5.1 it will be shown that only

$$
\left\langle\Delta_{1}^{1 / 3}, \Delta_{2}^{1 / 3}, \Delta_{3}^{1 / 3}\right\rangle_{0,3}^{P_{3,3}^{1}} \quad \text { and } \quad\left\langle\Delta_{i}^{1 / 3}, \Delta_{i}^{1 / 3}, \Delta_{i}^{1 / 3}\right\rangle_{0,3}^{1}
$$

for $i=1,2,3$ are nontrivial, which precisely give the coefficients $f_{0}$ and $f_{1}$ of cubic terms for the Gromov-Witten potential in [ST, Theorem 3.1].

\section{REMARK 5.1}

We will write the details on the classification orbispheres in $\mathbb{P}_{3,3,3}^{1}$ as concretely as possible. For the other two cases, $\mathbb{P}_{2,3,6}^{1}$ and $\mathbb{P}_{2,4,4}^{1}$, we will find similar classification results in Section 6, but without many details as the arguments are not all that different from the one for $\mathbb{P}_{3,3,3}^{1}$.

\subsection{Classification of orbispheres in $\mathbb{P}_{3,3,3}^{1}$}

From the expected dimension formula and the representability of an orbifold stable map, the only possible domain orbisphere in 0-dimensional components of the moduli space $\overline{\mathcal{M}}_{0,3, \beta}\left(\mathbb{P}_{3,3,3}^{1}\right)$ is $\mathbb{P}_{3,3,3}^{1}$ itself. Since there exists a unique biholomorphism $\phi:\left(\mathbb{P}_{3,3,3}^{1}, \boldsymbol{z}\right) \rightarrow\left(\mathbb{P}_{3,3,3}^{1}, \boldsymbol{z}^{\prime}\right)$ sending any triple of orbipoints $\boldsymbol{z}=\left(z_{1}, z_{2}, z_{3}\right)$ to another $\boldsymbol{z}^{\prime}=\left(z_{1}^{\prime}, z_{2}^{\prime}, z_{3}^{\prime}\right)$, there is no domain parameter in the relevant moduli $\overline{\mathcal{M}}_{0,3, \beta}\left(\mathbb{P}_{3,3,3}^{1}\right)$. Hence, from now on, we take the domain orbisphere to be $\mathbb{P}_{3,3,3}^{1}$ with the fixed conformal structure which is induced by the quotient map $E \rightarrow \mathbb{P}_{3,3,3}^{1}$ in Section 3.3.

By degree reason, $\left\langle\Delta_{\circ}^{i}, \Delta_{\bullet}^{j}, \Delta_{\diamond}^{k}\right\rangle_{0,3}^{\mathbb{P}_{3,3,3}^{1}}$ is trivial unless $i+j+k=1$. Note that, by the obvious symmetry on $\mathbb{P}_{3,3,3}^{1}$, it is enough to consider only the following three cases:

(1) $\left\langle\Delta_{1}^{1 / 3}, \Delta_{1}^{1 / 3}, \Delta_{1}^{1 / 3}\right\rangle_{0,3}$,

(2) $\left\langle\Delta_{1}^{1 / 3}, \Delta_{2}^{1 / 3}, \Delta_{3}^{1 / 3}\right\rangle_{0,3}$,

(3) $\left\langle\Delta_{1}^{1 / 3}, \Delta_{2}^{1 / 3}, \Delta_{2}^{1 / 3}\right\rangle_{0,3}$.

Namely, we may assume that the first marked point $z_{1}$ in the domain orbisphere is mapped to the orbisingular point $w_{1}$ in $\mathbb{P}_{3,3,3}^{1}$ associated with $\Delta_{1}^{1 / 3}$.

Let $u$ be a holomorphic orbisphere from $\mathbb{P}_{3,3,3}^{1}$ to $\mathbb{P}_{3,3,3}^{1}$ which contributes to $\left\langle\Delta_{1}^{1 / 3}, \Delta_{\circ}^{1 / 3}, \Delta_{\bullet}^{1 / 3}\right\rangle_{0,3}$. We fix base points of the domain orbisphere and the target orbisphere of $u$ and their universal coverings as follows: $x_{0}:=z_{1} \in \mathbb{P}_{3,3,3}^{1}$ and $\widetilde{x_{0}}:=0 \in p^{-1}(x)(\subset \mathbb{C})$ for the domain $\mathbb{P}_{3,3,3}^{1}$, and $y_{0}:=w_{1} \in \mathbb{P}_{3,3,3}^{1}$ and $\widetilde{y_{0}}:=$ $0 \in p^{-1}\left(w_{1}\right)(\subset \mathbb{C})$. Recall that $p: \mathbb{C} \rightarrow \mathbb{P}_{3,3,3}^{1}$ is the orbifold universal covering, and $u\left(x_{0}\right)=y_{0}$. Thus, we obtain a unique lifting $\tilde{u}$ of $u \circ p$ for the underlying 
holomorphic orbisphere $u: \mathbb{P}_{3,3,3}^{1} \rightarrow \mathbb{P}_{3,3,3}^{1}$ :

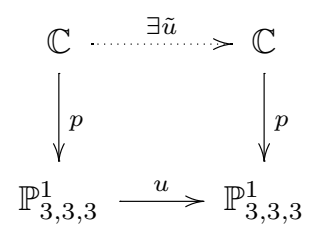

Note that the conditions in Lemma 4.2 are automatic in this case. Therefore, $u: \mathbb{P}_{3,3,3}^{1} \rightarrow \mathbb{P}_{3,3,3}^{1}$ is an orbifold covering, and its lifting $\tilde{u}$ from (5.1) has a particularly nice shape.

\section{PROPOSITION 5.2}

If $u$ is a nonconstant holomorphic orbisphere contributing to $\left\langle\Delta_{1}^{1 / 3}, \Delta_{\circ}^{1 / 3}, \Delta_{\bullet}^{1 / 3}\right\rangle_{0,3}$, then $\tilde{u}(z)=\lambda z$ for some $\lambda \in \mathbb{Z}[\tau]$, where $\mathbb{Z}[\tau]:=\{a+b \tau \mid a, b \in \mathbb{Z}\}$.

\section{Proof}

Because $u$ is an orbifold universal covering, so is the composition $u \circ p$. Now, by the uniqueness of orbifold universal coverings, $\tilde{u}$ should be a homeomorphism. Note that $\tilde{u}$ is an entire proper holomorphic map, since $\tilde{u}$ is a homeomorphism and is a lifting of the holomorphic map $u \circ p$. It is well known that any entire and proper holomorphic map on $\mathbb{C}$ is a polynomial. Since $\tilde{u}$ is invertible, we conclude that $\tilde{u}$ is a linear map $\tilde{u}(z)=\lambda z$ for some $\lambda \in \mathbb{C}$. Here, $\tilde{u}$ does not have a constant term because the lifting preserves the base points, $\tilde{u}\left(\widetilde{x_{0}}\right)=\widetilde{y_{0}}$ (i.e., $\left.\tilde{u}(0)=0\right)$.

Recall from Section 3.3 that $p^{-1}\left(x_{0}\right)$ gives a lattice $\mathbb{Z}\langle 1, \tau\rangle=\mathbb{Z}[\tau]\left(\because \tau^{2}=\right.$ $-\tau-1$ ) in $\mathbb{C} \cong \mathbb{R}^{2}$ (see the left-hand side of Figure 5 ). Since $1 \in \mathbb{C}$ is an element of this lattice, $\tilde{u}$ should map 1 to a point in $p^{-1}\left(y_{0}\right)$ which is also the same lattice $\mathbb{Z}[\tau]$ in $\mathbb{C}$. Thus, $\lambda=\tilde{u}(1)$ has to lie in $\mathbb{Z}[\tau]$, which finishes the proof.

It is obvious from the picture that the fundamental domain of the domain orbisphere covers that of the target orbisphere $|\lambda|^{2}$ times by the holomorphic map

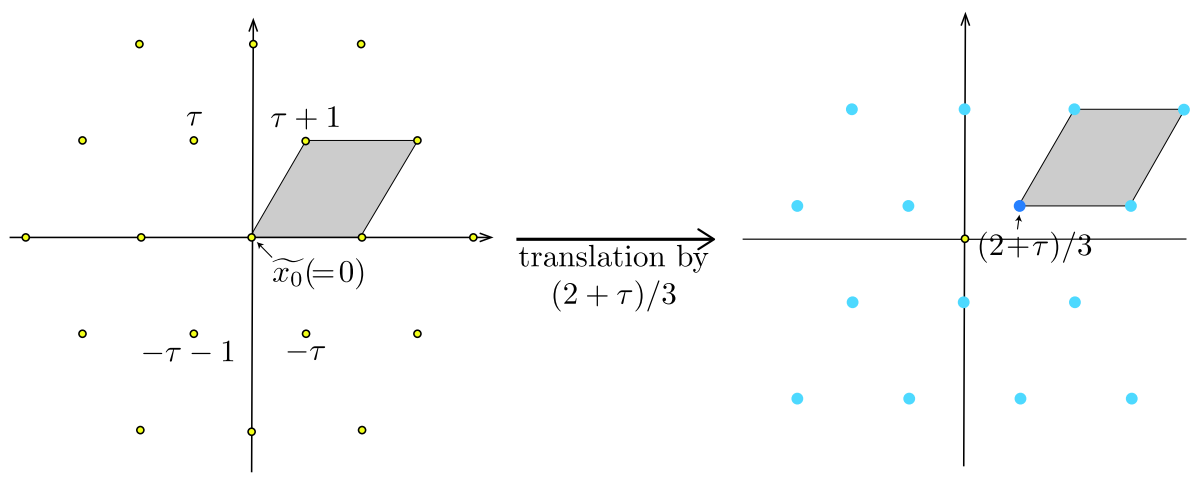

Figure 5. The shaded regions show the images of degree 3 maps contributing to $\left\langle\Delta_{1}^{1 / 3}, \Delta_{1}^{1 / 3}, \Delta_{1}^{1 / 3}\right\rangle_{0,3}$ (left-hand side) and $\left\langle\Delta_{2}^{1 / 3}, \Delta_{2}^{1 / 3}, \Delta_{2}^{1 / 3}\right\rangle_{0,3}$ (right-hand side). 
induced by the linear map $z \mapsto \lambda z$ between the universal covers. Another way to see this is to consider the energy $\int|d u|^{2}$ which equals the symplectic area of the holomorphic orbisphere $u$. Note that, for $\tilde{u}(z)=\lambda z,|d \tilde{u}|^{2}=|\lambda|^{2}$. Therefore, such a map induces a term containing $q^{|\lambda|^{2}}$ in the (3-point) Gromov-Witten potential.

Conversely, any linear map $\tilde{u}=\lambda z$ with a coefficient $\lambda$ in $\mathbb{Z}[\tau]$ induces a $\mathbb{Z}_{3^{-}}$ equivariant holomorphic map between the middle level torus $E$. The equivariance implies that $\tilde{u}$ descends to a holomorphic map $u: \mathbb{P}_{3,3,3}^{1} \rightarrow \mathbb{P}_{3,3,3}^{1}$. Then $u$ is a welldefined orbifold morphism between $\mathbb{P}_{3,3,3}^{1}$ 's, as it is represented by an equivariant map between $E$ 's, and $\mathbb{P}_{3,3,3}^{1}=\left[E / \mathbb{Z}_{3}\right]$. Later, we will establish the one-to-one correspondence between linear maps with $\mathbb{Z}[\tau]$-coefficients modulo certain equivalences and orbispheres in $\mathbb{P}_{3,3,3}^{1}$ contributing to $\left\langle\Delta_{1}^{1 / 3}, \Delta_{\circ}^{1 / 3}, \Delta_{\bullet}^{1 / 3}\right\rangle_{0,3}$ modulo equivalences given in (3.5).

\section{REMARK 5.3}

Let $\sigma$ be the cyclic permutation $(1,2,3)$ in the permutation group $S_{3}$ on three letters $\{1,2,3\}$. If $u$ contributes to $\left\langle\Delta_{1}^{1 / 3}, \Delta_{i}^{1 / 3}, \Delta_{j}^{1 / 3}\right\rangle_{0,3}$, then it is easy to see from Figure 5 that the translation of $\tilde{u}$ by an element of $\mathbb{Z}\left\langle\frac{2+\tau}{3}, \frac{1+2 \tau}{3}\right\rangle$ induces a map $v: \mathbb{P}_{3,3,3}^{1} \rightarrow \mathbb{P}_{3,3,3}^{1}$ contributing to $\left\langle\Delta_{\sigma^{k}(1)}^{1 / 3}, \Delta_{\sigma^{k}(i)}^{1 / 3}, \Delta_{\sigma^{k}(j)}^{1 / 3}\right\rangle_{0,3}$ for some $0 \leq$ $k \leq 2$. This explains the coincidence of various 3-point Gromov-Witten invariants appearing in [ST, Theorem 3.1]. Note that $\mathbb{Z}[\tau]$ is a sublattice of $\mathbb{Z}\left\langle\frac{2+\tau}{3}, \frac{1+2 \tau}{3}\right\rangle$.

\subsection{Symmetries of the lifting of orbimaps}

We have proved that any orbispheres with orbi-insertions $\Delta_{1}^{1 / 3}, \Delta_{i}^{1 / 3}, \Delta_{j}^{1 / 3}$ can be lifted to a linear map $z \mapsto \lambda z$ for $\lambda \in \mathbb{Z}[\tau]$. We next investigate a natural equivalence relation $\sim$ on $\{\tilde{u}=\lambda z \mid \lambda \in \mathbb{Z}[\tau]\}$ such that if $\tilde{u}_{1} \sim \tilde{u}_{2}$, then these maps induce a pair of equivalent holomorphic orbispheres. Let us denote the set of linear maps $\{z \mapsto \lambda z \mid \lambda \in \mathbb{Z}[\tau]\}$ by $L(\mathbb{C})$. We now find the equivalence relation on $L(\mathbb{C})$ such that the set of equivalence classes of $L(\mathbb{C})$ corresponds bijectively to the moduli space $\overline{\mathcal{M}}_{0,3}\left(\mathbb{P}_{3,3,3}^{1} ; \Delta_{1}^{1 / 3}, \Delta_{i}^{1 / 3}, \Delta_{j}^{1 / 3}\right)$ for $1 \leq i, j \leq 3$.

Recall that the positions of the three orbimarkings as well as the domain $\mathbb{P}_{3,3,3}^{1}$ itself are fixed by regarding $\mathbb{P}_{3,3,3}^{1}$ as a quotient of $E=\mathbb{C} / \mathbb{Z}\langle 1, \tau\rangle$ via the $\mathbb{Z} / 3$-action. Therefore, we do not have an equivalence from a domain reparameterization, and it is enough to find the condition for two linear maps $\tilde{u}_{i}(z)=\lambda_{i} z$ $\left(\lambda_{i} \in \mathbb{Z}[\tau],(i=1,2)\right)$ inducing the same map on the quotient orbifold.

Denote the induced orbispheres from $\tilde{u}_{i}$ by $u_{i}$ for $i=1,2$, and suppose that they have orbi-insertions $\Delta_{1}^{1 / 3}, \Delta_{i}^{1 / 3}$, and $\Delta_{j}^{1 / 3}$ at $z_{1}, z_{2}$, and $z_{3}$, respectively. Since $u_{1}$ and $u_{2}$ are the same orbifold morphism and both of them send $z_{1}$ to $w_{1}$, their local liftings should be related by the local isotropy group at $w_{1}$, which is isomorphic to $\mathbb{Z}_{3}$ and is generated by the $\tau$-multiplication. On the level of universal covers, this local group can be realized as the local isotropy group at the origin (lying in $p^{-1}\left(w_{1}\right)$ ) of $\mathbb{C}$. Local groups at other points in the fiber $p^{-1}\left(w_{i}\right)$ cannot relate $\tilde{u}_{1}$ and $\tilde{u}_{2}$ since they do not preserve the origin. Consequently, $\lambda_{1}=\tau^{k} \lambda_{2}$ for some $k=0,1,2$, and this gives the desired equivalence relation on $L(\mathbb{C})$. 
In summary, we have obtained an identification

$$
\left[L(\mathbb{C}) / \mathbb{Z}_{3}\right] \cong \bigcup_{i, j, d} \overline{\mathcal{M}}_{0,3, d}\left(\mathbb{P}_{3,3,3}^{1} ; \Delta_{1}^{1 / 3}, \Delta_{i}^{1 / 3}, \Delta_{j}^{1 / 3}\right)
$$

where the degree on the right-hand side corresponds to $|\lambda|^{2}=a^{2}-a b+b^{2}$ on the left-hand side (see the discussion below the proof of Proposition 5.2). In Section 5.3 it will be shown that the only possible $(i, j)$ 's are $(1,1),(2,3)$, and $(3,2)$.

For computational simplicity, we consider another type of symmetry on $\mathbb{P}_{3,3,3}^{1}$, which is induced from the $(1+\tau)$-multiplication on $\mathbb{C}$. (Note that $(1+\tau)^{2}=$ $\tau$.) This action gives rise to an action of $\mathbb{Z}_{6}$ on $L\left(\mathbb{C}\right.$ ) (and hence $\mathbb{Z}_{2}$-action on $\left.\left[L(\mathbb{C}) / \mathbb{Z}_{3}\right]\right)$ in an obvious way. In view of holomorphic orbispheres corresponding to elements of $L(\mathbb{C})$, this action switches two orbi-insertions $\Delta_{i}^{1 / 3}$ and $\Delta_{j}^{1 / 3}$ without changing the degree. Thus, for example, the $(1+\tau)$-multiplication gives rise to the one-to-one correspondence

$$
\overline{\mathcal{M}}_{0,3, d}\left(\mathbb{P}_{3,3,3}^{1} ; \Delta_{1}^{1 / 3}, \Delta_{2}^{1 / 3}, \Delta_{3}^{1 / 3}\right) \longleftrightarrow \overline{\mathcal{M}}_{0,3, d}\left(\mathbb{P}_{3,3,3}^{1} ; \Delta_{1}^{1 / 3}, \Delta_{3}^{1 / 3}, \Delta_{2}^{1 / 3}\right),
$$

both of which are components of the right-hand side of (5.2).

In Section 5.3, we will count elements in $L(\mathbb{C})$ whose underlying holomorphic orbispheres contribute $\left\langle\Delta_{1}^{1 / 3}, \Delta_{2}^{1 / 3}, \Delta_{3}^{1 / 3}\right\rangle_{0,3}$ or $\left\langle\Delta_{1}^{1 / 3}, \Delta_{3}^{1 / 3}, \Delta_{2}^{1 / 3}\right\rangle_{0,3}$ simultaneously. Then, dividing the number of such linear maps by the order of the group generated by the $(1+\tau)$-multiplication (which is 6 ), we find the presentation of

$$
f_{0}(q)=\sum_{d \in H_{2}(X, \mathbb{Z})}\left\langle\Delta_{1}^{1 / 3}, \Delta_{2}^{1 / 3}, \Delta_{3}^{1 / 3}\right\rangle_{0,3, d}^{X} q^{d} .
$$

\subsection{Identification of inputs}

We have shown that a degree $d$ nonconstant holomorphic orbisphere in $\mathbb{P}_{3,3,3}^{1}$ contributing to $\left\langle\Delta_{1}^{1 / 3}, \Delta_{i}^{1 / 3}, \Delta_{j}^{1 / 3}\right\rangle_{0,3, d}$ has a one-to-one correspondence with a linear map $z \mapsto \lambda z$ for some $\lambda=a+b \tau \in \mathbb{Z}[\tau]$ with $|\lambda|^{2}=d(\neq 0)$. (Recall that this $d$ is really the degree of the corresponding holomorphic orbisphere (see the discussion below the proof of Proposition 5.2).) We subdivide this set of holomorphic orbispheres in terms of their orbi-insertions. Orbi-insertions of the holomorphic orbisphere corresponding to $z \mapsto \lambda z$ can be determined in the following way. Note that the triangle with vertices $0, \frac{1+2 \tau}{3}$, and $\frac{2+\tau}{3}$ in the universal cover of the domain $\mathbb{P}_{3,3,3}^{1}$ gives a fundamental domain for the upper hemisphere of $\mathbb{P}_{3,3,3}^{1}$. Thus, we can think of $\frac{1+2 \tau}{3}$ and $\frac{2+\tau}{3}$ as (liftings of) the second and the third markings of the domain $\mathbb{P}_{3,3,3}^{1}$, respectively (see the shaded region on the left-hand side of Figure 6).

Since $\lambda \in \mathbb{Z}[\tau]$, the images $\lambda \cdot\left(\frac{1+2 \tau}{3}\right)$ and $\lambda \cdot\left(\frac{2+\tau}{3}\right)$ will lie in the lattice $\mathbb{Z}\left\langle\frac{1+2 \tau}{3}, \frac{2+\tau}{3}\right\rangle$ in the universal cover of the target $\mathbb{P}_{3,3,3}^{1}$. It is clear that the types of these two lattice points determine the orbi-insertions of the orbisphere associated with $\lambda$, that is, if $\lambda \cdot\left(\frac{1+2 \tau}{3}\right)$ lies in $\frac{1+2 \tau}{3}+\mathbb{Z}\langle 1, \tau\rangle$, then the second orbi-insertion is $\Delta_{2}^{1 / 3}$ and so on (see Figure 6 ). (Note that $z \mapsto \lambda z$ always sends the origin to 


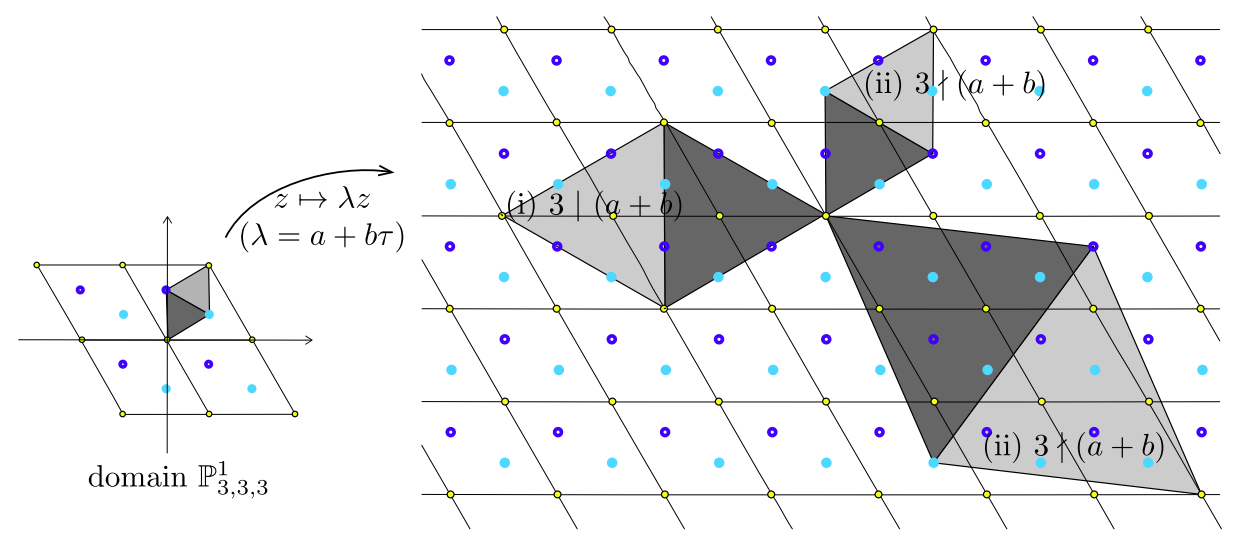

Figure 6. Images of holomorphic orbispheres in $\mathbb{P}_{3,3,3}^{1}$ visualized in its universal cover.

the origin, which is related to the fact that we fix the first orbi-insertion as $\Delta_{1}^{1 / 3}$ using the symmetry.)

Observe that

$$
\begin{aligned}
\lambda \cdot\left(\frac{1+2 \tau}{3}\right) & =(a+b \tau)\left(\frac{1+2 \tau}{3}\right) \\
& =\frac{(a-2 b)+(2 a-b) \tau}{3}
\end{aligned}
$$

and

$$
\lambda \cdot\left(\frac{2+\tau}{3}\right)=\frac{(2 a-b)+(a+b) \tau}{3} .
$$

(Here, we used the relation $\tau^{2}=-\tau-1$.) Using

$$
a-2 b \equiv a+b \quad \bmod 3,
$$

we see that there are only two possibilities:

(i) $3 \mid(a+b)$, for which both $\lambda \cdot\left(\frac{1+2 \tau}{3}\right)$ and $\lambda \cdot\left(\frac{2+\tau}{3}\right)$ correspond to the insertion $\Delta_{1}^{1 / 3}$;

(ii) $3 \nmid(a+b)$, for which both $\lambda \cdot\left(\frac{1+2 \tau}{3}\right)$ and $\lambda \cdot\left(\frac{2+\tau}{3}\right)$ correspond to two different insertions $\Delta_{2}^{1 / 3}$ and $\Delta_{3}^{1 / 3}$.

We remark that, in case (ii), insertions $\left(\Delta_{2}^{1 / 3}, \Delta_{3}^{1 / 3}\right)$ can be located at either $\left(z_{2}, z_{3}\right)$ or $\left(z_{3}, z_{2}\right)$ (see the discussion at the end of Section 5.2).

Note that

$$
d=|\lambda|^{2}=a^{2}-a b+b^{2}=(a+b)^{2}-3 a b \equiv(a+b)^{2} \quad \bmod 3 .
$$

Therefore, if $d \equiv 0 \bmod 3$, then the corresponding holomorphic spheres contribute to

$$
\left\langle\Delta_{1}^{1 / 3}, \Delta_{1}^{1 / 3}, \Delta_{1}^{1 / 3}\right\rangle_{0,3}
$$


and if $d \equiv 1 \bmod 3$, then they contribute to

$$
\left\langle\Delta_{1}^{1 / 3}, \Delta_{2}^{1 / 3}, \Delta_{3}^{1 / 3}\right\rangle_{0,3}
$$

Let $F(q)$ denote the power series

$$
F(q):=\sum_{a, b \in \mathbb{Z}} q^{a^{2}-a b+b^{2}} .
$$

See (A.1) for the first few terms of $F$.

By (5.3), the power of any nontrivial term in $F(q)$ should be either $0(\bmod 3)$ or $1(\bmod 3)$. Thus, we can decompose $F$ as $F=F_{0,3}+F_{1,3}$ according to the remainder of the power of $q$ when divided by 3 . Then the above discussion directly implies that

$$
f_{0}(q)=\sum_{d \in H_{2}(X, \mathbb{Z})}\left\langle\Delta_{1}^{1 / 3}, \Delta_{2}^{1 / 3}, \Delta_{3}^{1 / 3}\right\rangle_{0,3, d}^{X} q^{d}=\frac{1}{6} F_{1,3}(q)
$$

since there cannot be contributions from constant maps. Here, $\frac{1}{6}$ is responsible for the group $\mathbb{Z}_{6} \cong\langle 1+\tau\rangle$ which is discussed at the end of Section 5.2. For $f_{1}(q)=\sum_{d \in H_{2}(X, \mathbb{Z})}\left\langle\Delta_{1}^{1 / 3}, \Delta_{1}^{1 / 3}, \Delta_{1}^{1 / 3}\right\rangle_{0,3, d}^{X} q^{d}$, there is an additional contribution from the constant map (see Section $5.4,(5.6)$ ) so that $f_{1}(q)=\frac{1}{3} F_{0,3}(q)$, where $\frac{1}{3}$ again comes from $\mathbb{Z}_{3}$, the isotropy group at $w_{1}$ (or the origin in $\mathbb{C}$ ).

\section{REMARK 5.4}

Number-theoretic aspects of $F$ such as an explicit description of its Fourier coefficients will be given in Appendix A. In particular, we will describe the Fourier coefficients of $F$ in terms of the prime factorization of the exponent of $q$.

\subsection{Contribution from constant maps}

The constant map whose image lies in a single singular point also contributes to the quantum product. Indeed, these constant maps induce the product structure - of the Chen-Ruan [CR1] cohomology ring of $\mathbb{P}_{3,3,3}^{1}$, and the quantum product deforms this structure analogously to the relation between cup products and quantum products for smooth symplectic manifolds.

Let us consider one of the singular points $w_{i}$ and the constant maps from an orbisphere with three markings onto this point. The computation is essentially the same for all $i=1,2,3$ because of the symmetry. Obviously, there are two constant maps with image $w_{i}$ whose domain orbispheres are $\mathbb{P}_{3,3,3}^{1}$ and $\mathbb{P}_{3,3}^{1}$. We denote these maps by $c_{1}$ and $c_{2}$. Here, the markings for $c_{2}$ are located at two singular points and a chosen smooth point. We remark that the second map does not violate Lemma 4.1 since it only holds for nonconstant holomorphic orbispheres.

We have that $c_{1}$ and $c_{2}$ give rise to classical parts

$$
\left\langle\Delta_{i}^{1 / 3}, \Delta_{i}^{1 / 3}, \Delta_{i}^{1 / 3}\right\rangle_{0,3, d=0}^{X} \quad \text { and } \quad\left\langle\Delta_{i}^{1 / 3}, \Delta_{i}^{2 / 3}, 1\right\rangle_{0,3, d=0}^{X}
$$

of the 3-point Gromov-Witten invariant on $X=\mathbb{P}_{3,3,3}^{1}$. Both of these numbers are $\frac{1}{3}$, where the fraction comes from the definition of the orbifold integration 
(see [ALR] and Section 3.3). Therefore, the Chen-Ruan cup product for $\mathbb{P}_{3,3,3}^{1}$ is given as follows:

$$
\begin{aligned}
& \Delta_{i}^{1 / 3} \cdot \Delta_{i}^{2 / 3}=\frac{1}{3} P D(1)=\frac{1}{3}[\mathrm{pt}], \\
& \Delta_{i}^{1 / 3} \cdot \Delta_{i}^{1 / 3}=\frac{1}{3} P D\left(\Delta_{i}^{1 / 3}\right)=\Delta_{i}^{2 / 3} .
\end{aligned}
$$

Here, we used $P D\left(\Delta_{i}^{1 / 3}\right)=3 \times \Delta_{i}^{2 / 3}$ (see Remark 3.8), and (5.6) completes the computation of $f_{1}$.

\section{REMARK 5.5}

In fact, to verify (5.5), it remains to show that there are no other contributions than constants. However, we have shown in Lemma 4.1 that there are no holomorphic orbispheres in $\mathbb{P}_{3,3,3}^{1}$ which have only two orbifold markings.

\section{Further applications: $(2,3,6),(2,4,4)$}

In this section, we prove Theorem 1.2 and Proposition 1.3. We slightly modify the classification of holomorphic orbispheres in $\mathbb{P}_{3,3,3}^{1}$ in order to compute the quantum cohomology rings of the two other orbifold projective lines with three singular points: $\mathbb{P}_{2,3,6}^{1}$ and $\mathbb{P}_{2,4,4}^{1}$. For a certain product in $Q H_{\text {orb }}^{*}\left(\mathbb{P}_{2,3,6}^{1}\right)$, we use a heuristic argument, so the proof is incomplete (see Conjecture 6.3). In fact, all 3-point correlators including what remains as a conjecture here are computed in $[\mathrm{MR}]$. We hope to rediscover this missing part by classifying holomorphic orbispheres whose domain admits a hyperbolic structure, but we leave it to future investigation.

\subsection{The product on $Q H_{\text {orb }}^{*}\left(\mathbb{P}_{2,3,6}^{1}\right)$}

We set the notation for generators of $H_{\text {orb }}^{*}\left(\mathbb{P}_{2,3,6}^{1}\right)$ as follows. Recall that $E$ is the elliptic curve associated with the lattice $\mathbb{Z}\langle 1, \tau\rangle$ in $\mathbb{C}$ where $\tau=\exp \left(\frac{2 \pi \sqrt{-1}}{3}\right)$. Then $\mathbb{P}_{2,3,6}^{1}$ is obtained as the global quotient $\left[E / \mathbb{Z}_{6}\right]$, where $\mathbb{Z}_{6} \cong\langle 1+\tau\rangle$ acts on $E$ by complex multiplication. There are three cone points on $\mathbb{P}_{2,3,6}^{1}$ and we use the same notation $w_{1}, w_{2}$, and $w_{3}$ for these singular points as we did for $\mathbb{P}_{3,3,3}^{1}$, where $w_{1}, w_{2}$, and $w_{3}$ have isotropy groups $\mathbb{Z}_{2}, \mathbb{Z}_{3}$, and $\mathbb{Z}_{6}$, respectively. The inertia orbifold $\mathcal{I} \mathbb{P}_{2,3,6}^{1}$ consists of the smooth sectors $B \mathbb{Z}_{2}, B \mathbb{Z}_{3}$, and $B \mathbb{Z}_{6}$. The $\mathbb{Q}$-basis of $H_{\text {orb }}^{*}\left(\mathbb{P}_{2,3,6}^{1}, \mathbb{Q}\right)$ is given as $1, \Delta_{1}^{1 / 2}, \Delta_{2}^{1 / 3}, \Delta_{2}^{2 / 3}, \Delta_{3}^{1 / 6}, \ldots, \Delta_{3}^{5 / 6},[\mathrm{pt}]$ as follows.

The bases of the smooth sectors are

$$
H_{\text {orb }}^{0}\left(\mathbb{P}_{2,3,6}^{1}, \mathbb{Q}\right)=\mathbb{Q} \cdot 1, \quad H_{\text {orb }}^{2}\left(\mathbb{P}_{2,3,6}^{1}, \mathbb{Q}\right)=\mathbb{Q} \cdot[\mathrm{pt}] .
$$

For twist sectors, let $\Delta_{1}^{1 / 2} \in H_{\text {orb }}^{1}\left(\mathbb{P}_{2,3,6}^{1}, \mathbb{Q}\right), \Delta_{2}^{j / 3} \in H_{\text {orb }}^{\frac{2 j}{3}}\left(\mathbb{P}_{2,3,6}^{1}, \mathbb{Q}\right)(j=1,2)$, and $\Delta_{3}^{k / 6} \in H_{\mathrm{orb}}^{\frac{2 k}{6}}\left(\mathbb{P}_{2,3,6}^{1}, \mathbb{Q}\right)(k=1, \ldots, 5)$, which are supported at singular points $w_{1}$, $w_{2}$, and $w_{3}$, respectively. From the virtual dimension formula of $\overline{\mathcal{M}}_{0,3, d}\left(\mathbb{P}_{2,3,6}^{1}\right)$, 
we can classify all possible orbi-insertions with expected dimension 0 and the corresponding domain orbisphere as in the following list:

(a) $\mathbb{P}_{2,3,6}^{1}:\left\langle\Delta_{1}^{1 / 2}, \Delta_{2}^{1 / 3}, \Delta_{3}^{1 / 6}\right\rangle,\left\langle\Delta_{3}^{3 / 6}, \Delta_{2}^{1 / 3}, \Delta_{3}^{1 / 6}\right\rangle,\left\langle\Delta_{1}^{1 / 2}, \Delta_{3}^{2 / 6}, \Delta_{3}^{1 / 6}\right\rangle,\left\langle\Delta_{3}^{3 / 6}\right.$, $\left.\Delta_{3}^{2 / 6}, \Delta_{3}^{1 / 6}\right\rangle$

(b) $\mathbb{P}_{3,3,3}^{1}:\left\langle\Delta_{3}^{2 / 6}, \Delta_{3}^{2 / 6}, \Delta_{3}^{2 / 6}\right\rangle,\left\langle\Delta_{2}^{1 / 3}, \Delta_{3}^{2 / 6}, \Delta_{3}^{2 / 6}\right\rangle,\left\langle\Delta_{2}^{1 / 3}, \Delta_{2}^{1 / 3}, \Delta_{3}^{2 / 6}\right\rangle,\left\langle\Delta_{2}^{1 / 3}\right.$, $\left.\Delta_{2}^{1 / 3}, \Delta_{2}^{1 / 3}\right\rangle$

(c) $\mathbb{P}_{3,6,6}^{1}$ (hyperbolic): $\left\langle\Delta_{3}^{1 / 6}, \Delta_{3}^{1 / 6}, \Delta_{3}^{4 / 6}\right\rangle,\left\langle\Delta_{2}^{2 / 3}, \Delta_{3}^{1 / 6}, \Delta_{3}^{1 / 6}\right\rangle$;

(d) $\mathbb{P}_{2,2}^{1}:\left\langle 1, \Delta_{3}^{3 / 6}, \Delta_{3}^{3 / 6}\right\rangle,\left\langle 1, \Delta_{1}^{1 / 2}, \Delta_{3}^{3 / 6}\right\rangle,\left\langle 1, \Delta_{1}^{1 / 2}, \Delta_{1}^{1 / 2}\right\rangle$;

(e) $\mathbb{P}_{3,3}^{1}:\left\langle 1, \Delta_{2}^{1 / 3}, \Delta_{3}^{4 / 6}\right\rangle,\left\langle 1, \Delta_{2}^{2 / 3}, \Delta_{3}^{2 / 6}\right\rangle$.

From Lemma 4.1, there are no nontrivial maps which contribute to types (d) and (e). Thus, if we denote $t:=\sum t_{j, i} \Delta_{j}^{i}$, then the genus 0 Gromov-Witten potential of $\mathbb{P}_{2,3,6}^{1}$ can be written up to order $t^{3}$ as follows:

$$
\begin{aligned}
F_{0}^{\mathbb{P}_{2,3,6}^{1}}(\boldsymbol{t})= & \frac{1}{2} t_{0}^{2} \log q+t_{0}\left(\frac{1}{2} t_{1, \frac{1}{2}} t_{1, \frac{1}{2}}+\frac{1}{6} t_{3, \frac{3}{6}} t_{3, \frac{3}{6}}\right)+\left(t_{1, \frac{1}{2}} t_{2, \frac{1}{3}} t_{3, \frac{1}{6}}\right) \cdot h_{0}(q) \\
& +\left(t_{3, \frac{3}{6}} t_{2, \frac{1}{3}} t_{3, \frac{1}{6}}\right) \cdot h_{1}(q)+\left(t_{1, \frac{1}{2}} t_{3, \frac{2}{6}} t_{3, \frac{1}{6}}\right) \cdot h_{2}(q)+\left(t_{3, \frac{3}{6}} t_{3, \frac{2}{6}} t_{3, \frac{1}{6}}\right) \cdot h_{3}(q) \\
(6.1) \quad & \frac{1}{6} t_{3, \frac{2}{6}}^{3} \cdot h_{4}(q)+\frac{1}{2} t_{3, \frac{2}{6}}^{2} t_{2, \frac{1}{3}} \cdot h_{5}(q)+\frac{1}{2} t_{3, \frac{2}{6}} t_{2, \frac{1}{3}}^{2} \cdot h_{6}(q)+\frac{1}{6} t_{2, \frac{1}{3}}^{3} \cdot h_{7}(q) \\
& +\frac{1}{2} t_{3, \frac{1}{6}}^{2} t_{3, \frac{4}{6}} \cdot h_{8}(q)+\frac{1}{2} t_{3, \frac{1}{6}}^{2} t_{2, \frac{2}{3}} \cdot h_{9}(q)+\frac{1}{2} t_{2, \frac{2}{3}} t_{3, \frac{1}{6}}^{2} \cdot h_{10}(q)+O\left(t^{4}\right),
\end{aligned}
$$

where the precise expressions of $h_{i}(q)$ for $0 \leq i \leq 10$ will be given later.

For holomorphic orbispheres of types (a) and (b), we choose the presentations of domain orbispheres as $\left[E / \mathbb{Z}_{6}\right]$ and $\left[E / \mathbb{Z}_{3}\right]$, respectively. Here, $E$ is the elliptic curve corresponding to the $\mathbb{Z}$-lattice $\langle 1, \tau\rangle$ in $\mathbb{C}$, where $\tau=\exp \frac{2 \pi \sqrt{-1}}{3}$.

Observe that any holomorphic orbisphere with the orbi-insertion condition in (a) or (b) satisfies the condition in Lemma 4.2. So, we can lift such maps $u$ : $\mathbb{P}_{2,3,6}^{1} \rightarrow \mathbb{P}_{2,3,6}^{1}$ and $u: \mathbb{P}_{3,3,3}^{1} \rightarrow \mathbb{P}_{2,3,6}^{1}$ to a linear map between universal coverings $\tilde{u}: \mathbb{C} \rightarrow \mathbb{C}$. Below, we will count these holomorphic orbispheres with the help of the lattice structures of the inverse image of orbisingular points in $\mathbb{C}$. As in the case of $\mathbb{P}_{3,3,3}^{1}$, it will be shown that the counting matches the number of solutions of certain Diophantine equations. The regularity of these holomorphic orbispheres is guaranteed by Lemma 4.4 .

To clarify the orbi-insertions by looking at the lifted linear map $\tilde{u}: \mathbb{C} \rightarrow \mathbb{C}$, we explicitly identify the lattice structure on $\mathbb{C}$ coming from the universal orbifold covering map $p: \mathbb{C} \rightarrow \mathbb{P}_{2,3,6}^{1}$ as follows:

$$
\begin{aligned}
p^{-1}\left(w_{1}\right) & =\mathbb{Z}\left\langle\frac{1}{2}, \frac{\tau}{2}\right\rangle+p^{-1}\left(w_{3}\right) \\
& =\left\{\frac{a}{2}+\frac{b}{2} \tau \mid a, b \in \mathbb{Z} \text { and } a \text { or } b \text { is an odd integer }\right\}, \\
p^{-1}\left(w_{2}\right) & =\mathbb{Z}\left\langle\frac{2+\tau}{3}, \frac{1+2 \tau}{3}\right\rangle+p^{-1}\left(w_{1}\right), \\
p^{-1}\left(w_{3}\right) & =\mathbb{Z}\langle 1, \tau\rangle \quad(\ni 0) .
\end{aligned}
$$




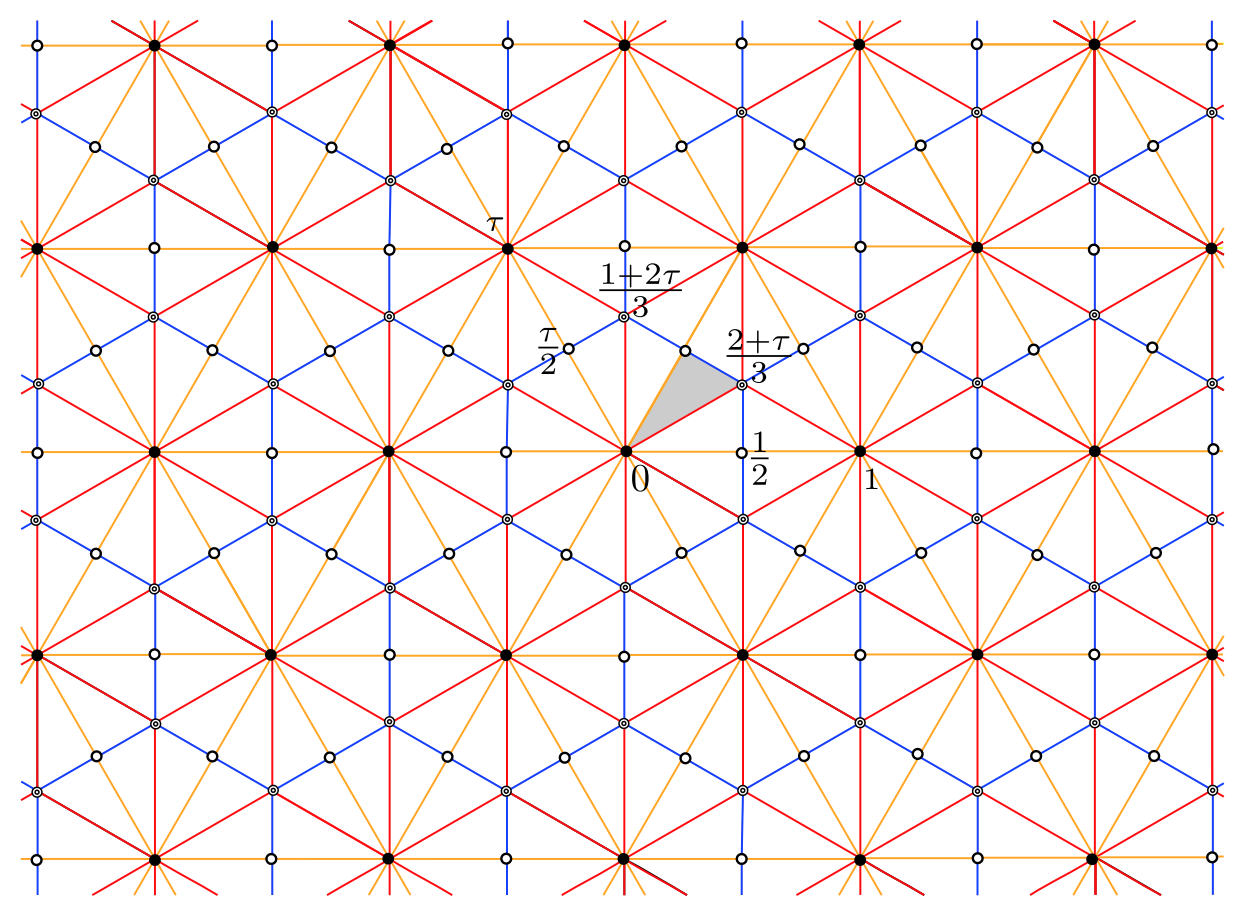

Figure 7. Lattices on the universal cover of $\mathbb{P}_{2,3,6}^{1}: p^{-1}\left(w_{1}\right)=\{\circ\}, p^{-1}\left(w_{2}\right)=\{\odot\}$, and $p^{-1}\left(w_{3}\right)=\{\bullet\}$.

In particular, $w_{3}$ is set to be a base point associated with the universal covering $(\mathbb{C}, 0)$ (see Figure 7).

The universal cover $\mathbb{C}$ of the domain $\mathbb{P}_{2,3,6}^{1}$ also has the same lattice structure, and the lattices on $\mathbb{C}$ from the domain $\mathbb{P}_{3,3,3}^{1,3}$ are given as in Section 5.3.

Case (a) with the domain orbisphere $\mathbb{P}_{2,3,6}^{1}: h_{i}$ for $0 \leq i \leq 3$

Let $z_{1}, z_{2}$, and $z_{3}$ be the three orbipoints in the domain $\mathbb{P}_{2,3,6}^{1}$ whose orders of singularity are 2,3 , and 6 , respectively. If $u$ is a holomorphic map from $\mathbb{P}_{2,3,6}^{1}$ to itself with the orbi-insertion condition as in (a), then $u$ is an orbifold covering map by Lemma 4.2 , so one can find the lifting $\tilde{u}: \mathbb{C} \rightarrow \mathbb{C}$ with $\tilde{u}(0)=0$ :

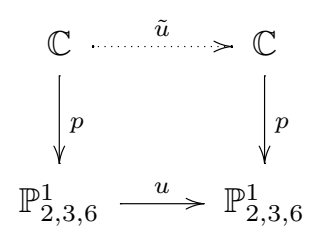

Since any holomorphic orbisphere $u$ contributing to (a) maps $z_{3}$ to $w_{3}$ (by the arrangement of insertions in (a)), $\tilde{u}(z)=\lambda z$ for some $\lambda \in \mathbb{Z}[\tau]$. Conversely, it is clear from Figure 7 that any such linear map $\tilde{u}$ descends to a holomorphic orbisphere with insertions as in (a). Since for $\lambda=a+b \tau(a, b \in \mathbb{Z})$, the degree of the underlying map of $\tilde{u}(z)=\lambda z$ is $N:=|\lambda|^{2}=\lambda \bar{\lambda}=a^{2}-a b+b^{2}$, the above 
discussion shows that

$$
h_{0}(q)+h_{1}(q)+h_{2}(q)+h_{3}(q)=\frac{1}{6} F(q),
$$

where $F(q)$ is defined by (5.4). Here, $\frac{1}{6}$ on the right-hand side comes from the symmetry between linear maps which induce the same holomorphic orbisphere. By the same argument as in Section 5.2, we see that the symmetry among these linear maps is generated by $(1+\tau)$-multiplication, which is nothing but the action of the isotropy group of $w_{3}$ (isomorphic to $\mathbb{Z}_{6}$ ).

Note that the triangle, whose vertices are $0 \in p^{-1}\left(z_{3}\right), \frac{2+\tau}{3} \in p^{-1}\left(z_{2}\right)$, and $\frac{1+\tau}{2} \in p^{-1}\left(z_{1}\right)$, gives the fundamental domain of the upper hemisphere of (the domain) $\mathbb{P}_{2,3,6}^{1}$ (see the shaded region in Figure 7 and compare it with Figure 8). As in the case of $\mathbb{P}_{3,3,3}^{1}$, we classify the orbi-insertion condition by chasing the images of $\frac{2+\tau}{3}$ and $\frac{1+\tau}{2}$ in the domain. For $\lambda=a+b \tau$,

$$
\lambda \cdot \frac{2+\tau}{3}=\frac{2 a-b}{3}+\frac{a+b}{3} \tau
$$

and

$$
\lambda \cdot \frac{1+\tau}{2}=\frac{a-b}{2}+\frac{a}{2} \tau
$$

First, note that

$$
\begin{aligned}
& \lambda \cdot \frac{1+\tau}{2} \in p^{-1}\left(w_{1}\right) \Longleftrightarrow \quad a \text { or } b \text { is odd, } \\
& \lambda \cdot \frac{1+\tau}{2} \in p^{-1}\left(w_{3}\right) \quad \Longleftrightarrow \quad a \text { and } b \text { is even. }
\end{aligned}
$$

From $3|2 a-b \Longleftrightarrow 3| a+b$, it can be easily checked that

$$
\begin{aligned}
\lambda \cdot \frac{2+\tau}{3} \in p^{-1}\left(w_{3}\right) & \Longleftrightarrow 3 \mid(a+b), \\
\lambda \cdot \frac{2+\tau}{3} \in p^{-1}\left(w_{2}\right) & \Longleftrightarrow 3 \nmid(a+b) .
\end{aligned}
$$

Hence, using (5.3), we see that there are two possible orbi-insertions at the marked point corresponding to $\lambda \cdot \frac{2+\tau}{3}$ :

$\Delta_{3}^{2 / 6} 3 \mid(a+b) \Longleftrightarrow N \equiv 0 \bmod 3$,

$\Delta_{2}^{1 / 3} 3 \nmid(a+b) \Longleftrightarrow N \equiv 1 \bmod 3$.

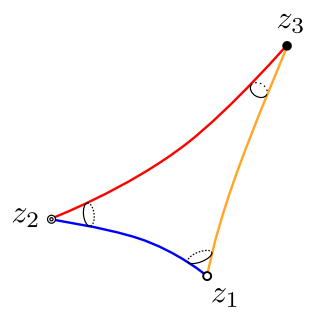

Figure 8 . $\mathbb{P}_{2,3,6}^{1}$. 
Similarly, two possible orbi-insertions at the marked point corresponding to $\lambda$. $\frac{1+\tau}{2}$ are

$\Delta_{3}^{3 / 6} a$ and $b$ is even $\Longleftrightarrow N \equiv 0 \bmod 2$,

$\Delta_{1}^{1 / 2} a$ or $b$ is odd $\Longleftrightarrow N \equiv 1 \bmod 2$.

Summarizing the above discussion, we conclude from the Chinese remainder theorem that $u$ contributes to

$$
\begin{aligned}
& \left\langle\Delta_{1}^{1 / 2}, \Delta_{2}^{1 / 3}, \Delta_{3}^{1 / 6}\right\rangle \Longleftrightarrow \operatorname{deg} u \equiv 1 \bmod 6, \\
& \left\langle\Delta_{3}^{3 / 6}, \Delta_{2}^{1 / 3}, \Delta_{3}^{1 / 6}\right\rangle \Longleftrightarrow \operatorname{deg} u \equiv 4 \bmod 6, \\
& \left\langle\Delta_{1}^{1 / 2}, \Delta_{3}^{2 / 6}, \Delta_{3}^{1 / 6}\right\rangle \Longleftrightarrow \operatorname{deg} u \equiv 3 \bmod 6, \\
& \left\langle\Delta_{3}^{3 / 6}, \Delta_{3}^{2 / 6}, \Delta_{3}^{1 / 6}\right\rangle \Longleftrightarrow \operatorname{deg} u \equiv 0 \bmod 6 .
\end{aligned}
$$

Recall $N=|\lambda|^{2}=\operatorname{deg} u$, which equals the exponent of $q$ for the term in the Gromov-Witten potential to which $u$ contributes. Therefore, we obtain

$$
\begin{aligned}
& h_{0}(q)=\frac{1}{6} \sum_{\substack{N=1 \\
N \equiv 1 \bmod 6}}^{\infty} \sum_{\substack{m n+n^{2}=N \\
m, n \in \mathbb{Z}}} q^{N}=\frac{1}{6} F_{1,6}(q), \\
& h_{1}(q)=\frac{1}{6} \sum_{\substack{N=1 \\
N \equiv 4 \bmod 6}}^{\infty} \sum_{\substack{m^{2} \\
m}} q^{N}=\frac{1}{6} F_{4,6}(q), \\
& h_{2}(q)=\frac{1}{6} \sum_{\substack{N=1 \\
m, n \in \mathbb{Z}}}^{\infty} \sum_{\substack{\text { mod } 6 \\
m^{2}-\underset{m n+n^{2}}{m, n \in \mathbb{Z}}=N}} q^{N}=\frac{1}{6} F_{3,6}(q), \\
& h_{3}(q)=\frac{1}{6}\left(1+\sum_{\substack{N=1 \\
N \equiv 0 \bmod 6}}^{\infty} \sum_{\substack{m^{2}-m_{m,+n^{2}}=N \\
m, n \in \mathbb{Z}}}^{\infty} q^{N}\right)=\frac{1}{6}+\frac{1}{6} F_{0,6}(q),
\end{aligned}
$$

where $F_{i, 6}$ is the sum of terms in $F$ whose exponents of $q$ are $i$ modulo 6 . Here, the constant term of $h_{3}$ can be obtained from a similar argument in Section 5.4.

Case (b) with the domain orbisphere $\mathbb{P}_{3,3,3}^{1}$

We first show that holomorphic orbispheres with orbi-insertions as in case (b) can be lifted to the one on $\mathbb{P}_{3,3,3}^{1}$. Let $\pi: \mathbb{P}_{3,3,3}^{1} \rightarrow \mathbb{P}_{2,3,6}^{1}$ be the 2 -fold orbifold covering map which comes from the action on $[E /\langle\tau\rangle]$ generated by $(1+\tau)$-multiplication, as drawn in Figure 9. Write $w_{i}^{\prime}$ and $w_{i}(i=1,2,3)$ for orbipoints in $\mathbb{P}_{3,3,3}^{1}$ and $\mathbb{P}_{2,3,6}^{1}$, respectively, and let $\pi$ send both $w_{1}^{\prime}$ and $w_{2}^{\prime}$ to $w_{2}$ and send $w_{3}^{\prime}$ to $w_{3}$.

After fixing base points of $\mathbb{P}_{3,3,3}^{1}$ and $\mathbb{P}_{2,3,6}^{1}$, we have (see Section 2.2)

$$
\begin{aligned}
& \pi_{1}^{\text {orb }}\left(\mathbb{P}_{3,3,3}^{1}\right)=\left\langle\rho_{1}, \rho_{2}, \rho_{3} \mid\left(\rho_{1}\right)^{3}=\left(\rho_{2}\right)^{3}=\left(\rho_{3}\right)^{3}=\rho_{1} \rho_{2} \rho_{3}=1\right\rangle, \\
& \pi_{1}^{\text {orb }}\left(\mathbb{P}_{2,3,6}^{1}\right)=\left\langle\lambda_{1}, \lambda_{2}, \lambda_{3} \mid\left(\lambda_{1}\right)^{2}=\left(\lambda_{2}\right)^{3}=\left(\lambda_{3}\right)^{6}=\lambda_{1} \lambda_{2} \lambda_{3}=1\right\rangle,
\end{aligned}
$$

and $\pi$ induces a group homomorphism $\pi_{*}: \pi_{1}^{\text {orb }}\left(\mathbb{P}_{3,3,3}^{1}\right) \rightarrow \pi_{1}^{\text {orb }}\left(\mathbb{P}_{2,3,6}^{1}\right)$. We see from Figure 9 that the images of $\rho_{1}$ and $\rho_{2}$ under $\pi_{*}$ lie in the conjugacy class of 


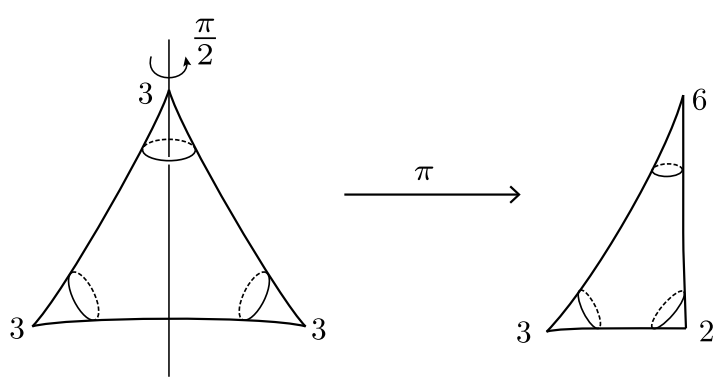

Figure 9. The 2-fold covering $\pi: \mathbb{P}_{3,3,3}^{1} \rightarrow \mathbb{P}_{2,3,6}^{1}$.

$\lambda_{2}$, and the image of the other generator $\rho_{3}$ lies in that of $\left(\lambda_{3}\right)^{2}$. (Here, conjugacy classes depend on the choice of base points.) It follows that $\pi_{*}\left(\pi_{1}^{\text {orb }}\left(\mathbb{P}_{3,3,3}^{1}\right)\right)$ contains $\lambda_{2}$ and $\left(\lambda_{3}\right)^{2}$, as it is a normal subgroup of $\pi_{1}^{\text {orb }}\left(\mathbb{P}_{2,3,6}^{1}\right)$.

LEMMA 6.1

For a given (b)-type holomorphic orbisphere $u: \mathbb{P}_{3,3,3}^{1} \rightarrow \mathbb{P}_{2,3,6}^{1}$, there exists a holomorphic orbisphere $\tilde{u}$ which makes the following diagram commute:

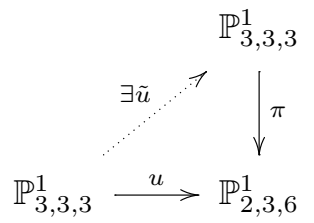

\section{Proof}

Observe that only two kinds of orbi-insertions $\Delta_{2}^{1 / 3}$ and $\Delta_{3}^{2 / 6}$ appear in (b). Hence $u_{*}$ maps a generator of $\pi_{1}^{\text {orb }}\left(\mathbb{P}_{3,3,3}^{1}\right)$ to an element in the conjugacy class of $\lambda_{2}$ or $\lambda_{3}^{2}$. (Indeed, if we choose base points and the generators $\rho_{1}$ and $\lambda_{2}$ as in Figure $1(\mathrm{~b})$, then $u_{*}$ sends $\rho_{1}$ exactly to $\lambda_{2}$, and a similar result follows for $\rho_{2}$ and $\rho_{3}$.) Thus, $u_{*}\left(\pi_{1}^{\text {orb }}\left(\mathbb{P}_{3,3,3}^{1}\right)\right)$ is contained in $\pi_{*}\left(\pi_{1}^{\text {orb }}\left(\mathbb{P}_{3,3,3}^{1}\right)\right)$. From Proposition 2.8, there exists an orbimap $\tilde{u}: \mathbb{P}_{3,3,3}^{1} \rightarrow \mathbb{P}_{3,3,3}^{1}$ which lifts $u$.

Let $z$ be one of the three orbipoints of the domain $\mathbb{P}_{3,3,3}^{1}$ for a holomorphic orbisphere $u$ of type (b). If $u$ sends $z$ to $w_{2} \in \mathbb{P}_{2,3,6}^{1}$ with the orbi-insertion $\Delta_{2}^{1 / 3}$, then the corresponding orbi-insertion of the lifting $\tilde{u}: \mathbb{P}_{3,3,3}^{1} \rightarrow \mathbb{P}_{3,3,3}^{1}$ is $\Delta_{1}^{1 / 3}$ or $\Delta_{2}^{1 / 3}$. Similarly, if $u(z)=w_{3}$ with the insertion $\Delta_{3}^{2 / 6}$, then the corresponding orbiinsertion of a lifting $\tilde{u}$ is $\Delta_{3}^{1 / 3}$. Here, we abused the notation for orbi-insertions of $\mathbb{P}_{3,3,3}^{1}$ and $\mathbb{P}_{2,3,6}^{1}$.

For each holomorphic orbisphere $u: \mathbb{P}_{3,3,3}^{1} \rightarrow \mathbb{P}_{2,3,6}^{1}$ with orbi-insertion of type (b), there are two liftings $\tilde{u}: \mathbb{P}_{3,3,3}^{1} \rightarrow \mathbb{P}_{3,3,3}^{1}$. Two liftings of $u$ are related by the $\mathbb{Z}_{2}$-action (i.e., the action of the deck transformation group) which switches $w_{1}^{\prime}$ and $w_{2}^{\prime}$. Therefore, if one lifting has orbi-insertion $\Delta_{1}^{1 / 3}$, then the other lifting has 
orbi-insertion $\Delta_{2}^{1 / 3}$. In summary, Lemma 6.1 gives rise to the following one-to-two correspondences:

$$
\begin{aligned}
& \left\langle\Delta_{3}^{2 / 6}, \Delta_{3}^{2 / 6}, \Delta_{3}^{2 / 6}\right\rangle^{\mathbb{P}_{2,3,6}^{1}} \stackrel{1: 2}{\longleftrightarrow}\left\langle\Delta_{3}^{1 / 3}, \Delta_{3}^{1 / 3}, \Delta_{3}^{1 / 3}\right\rangle^{1} \mathbb{P}_{3,3,3}, \\
& \left\langle\Delta_{2}^{1 / 3}, \Delta_{2}^{1 / 3}, \Delta_{3}^{2 / 6}\right\rangle^{\mathbb{P}_{2,3,6}^{1}} \stackrel{1: 2}{\longleftrightarrow}\left\langle\Delta_{1}^{1 / 3}, \Delta_{2}^{1 / 3}, \Delta_{3}^{1 / 3}\right\rangle^{1}{ }^{1,3,3}+\left\langle\Delta_{2}^{1 / 3}, \Delta_{1}^{1 / 3}, \Delta_{3}^{1 / 3}\right\rangle^{\mathbb{P}_{3,3,3}^{1}} \\
& =2\left\langle\Delta_{1}^{1 / 3}, \Delta_{2}^{1 / 3}, \Delta_{3}^{1 / 3}\right\rangle_{3,3,3}^{1}, \\
& \left\langle\Delta_{2}^{1 / 3}, \Delta_{2}^{1 / 3}, \Delta_{2}^{1 / 3}\right\rangle^{\mathbb{P}_{2,3,6}^{1}} \stackrel{1: 2}{\longleftrightarrow}\left\langle\Delta_{1}^{1 / 3}, \Delta_{1}^{1 / 3}, \Delta_{1}^{1 / 3}\right\rangle^{\mathbb{P}_{3,3,3}^{1}}+\left\langle\Delta_{2}^{1 / 3}, \Delta_{2}^{1 / 3}, \Delta_{2}^{1 / 3}\right\rangle^{\mathbb{P}_{3,3,3}^{1}} \\
& =2\left\langle\Delta_{1}^{1 / 3}, \Delta_{1}^{1 / 3}, \Delta_{1}^{1 / 3}\right\rangle_{3,3,3}^{1} .
\end{aligned}
$$

(Since there are no corresponding liftings, $\left\langle\Delta_{2}^{1 / 3}, \Delta_{3}^{2 / 6}, \Delta_{3}^{2 / 6}\right\rangle^{\mathbb{P}_{2,3,6}^{1}}$ vanishes.)

Therefore, $h_{i}$ for $4 \leq i \leq 7$ is given as follows.

\section{PROPOSITION 6.2}

Let $f_{0}^{\mathbb{P}_{3,3,3}^{1}}(q)$ and $f_{1}^{\mathbb{P}_{3,3,3}^{1}}(q)$ be the coefficients of $t_{1} t_{2} t_{3}$ and $t_{i}^{3}$ of $F_{0}^{\mathbb{P}_{3,3,3}^{1}}$, respectively. Then

$$
\begin{aligned}
& h_{4}(q)=\frac{1}{2} f_{1}^{\mathbb{P}_{3,3,3}^{1}}\left(q^{2}\right)=\frac{1}{6}+q^{6}+q^{18}+q^{24}+2 q^{42}+O\left(q^{48}\right), \\
& h_{5}(q)=0 \\
& h_{6}(q)=f_{0}^{\mathbb{P}_{3,3,3}^{1}}\left(q^{2}\right)=q^{2}+q^{8}+2 q^{14}+2 q^{26}+q^{32}+2 q^{38}+O\left(q^{48}\right), \\
& h_{7}(q)=f_{1}^{\mathbb{P}_{3,3,3}^{1}}\left(q^{2}\right)=\frac{1}{3}+2 q^{6}+2 q^{18}+2 q^{24}+4 q^{42}+O\left(q^{48}\right) .
\end{aligned}
$$

Case (c) with the domain orbisphere $\mathbb{P}_{3,6,6}^{1}$

For these kinds of contributions, the lifting of holomorphic orbispheres on the universal cover level is no longer a linear map, since the domain orbisphere is hyperbolic. Hence, we cannot use our classification argument any longer. However, we may try to find such maps directly by looking at their image on the universal cover $\mathbb{C}$ of the target $\mathbb{P}_{2,3,6}^{1}$.

For this, we consider rhombi in the universal covering of $\mathbb{P}_{2,3,6}^{1}$ whose vertices lie in the $p^{-1}\left(w_{1}, w_{2}, w_{3}\right)$. For example, observe that the rhombus $v$ whose set of vertices are $\left\{0, \frac{2+\tau}{3}, 1, \frac{1-\tau}{3}\right\}$ gives one contribution from $\mathbb{P}_{3,6,6}^{1}$ to $\left\langle\Delta_{2}^{2 / 3}\right.$, $\left.\Delta_{3}^{1 / 6}, \Delta_{2}^{1 / 6}\right\rangle$ (see the rightmost rhombus in Figure 10). One can visualize this holomorphic orbisphere by folding this rhombus along its longer diagonal. Pairs of identified edges after this process are drawn in Figure 10.

There are various such rhombi, and their corresponding orbi-insertions can be classified in the following way. Note that these rhombi are images of the smallest rhombus $v$ given above by linear maps $z \mapsto \lambda z$ for $\lambda \in \mathbb{Z}[\tau]$. (This means that we regard the vertices $\frac{2+\tau}{3}$ and $\frac{1-\tau}{3}$ of $v$ as markings $z_{2}$ and $z_{3}$ of order 6 in the domain $\mathbb{P}_{3,6,6}^{1}$, respectively.) Since $(1+\tau) \cdot \frac{1-\tau}{3}=\frac{2+\tau}{3}$, insertions at $z_{2}$ and $z_{3}$ are the same, and if $\lambda \cdot\left(\frac{2+\tau}{3}\right)$ is contained in $p^{-1}\left(w_{3}\right)$ (resp., $p^{-1}\left(w_{2}\right)$ ), then 


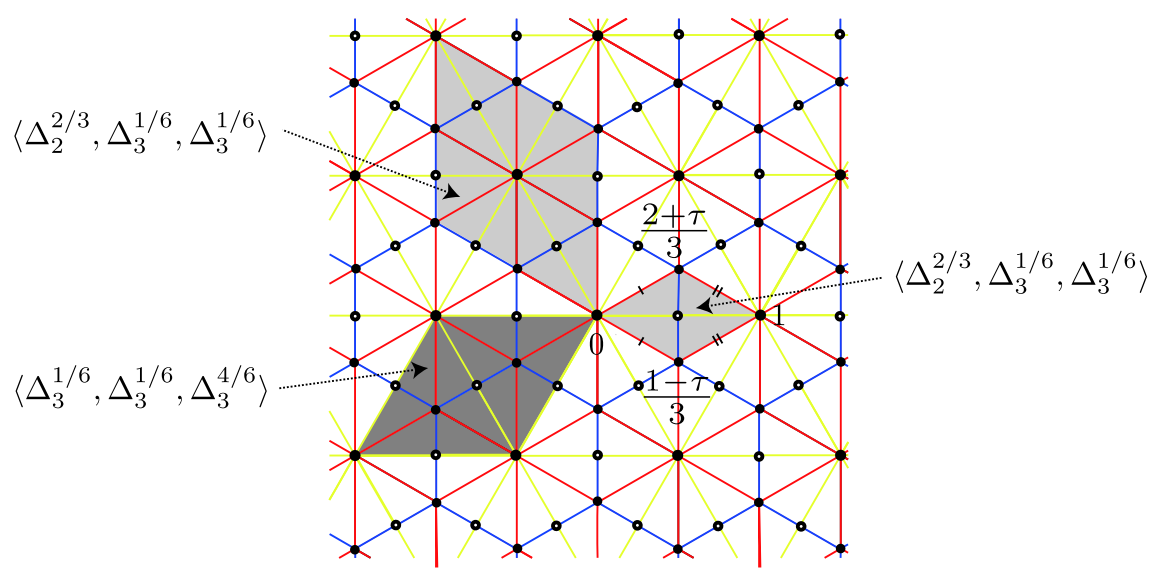

Figure 10. Images of holomorphic orbispheres $\mathbb{P}_{3,6,6}^{1} \rightarrow \mathbb{P}_{2,3,6}^{1}$ visualized in the universal cover of $\mathbb{P}_{2,3,6}^{1}$.

the corresponding insertions are $\left\langle\Delta_{3}^{1 / 6}, \Delta_{3}^{1 / 6}, \Delta_{3}^{4 / 6}\right\rangle$ (resp., $\left\langle\Delta_{2}^{2 / 3}, \Delta_{3}^{1 / 6}, \Delta_{3}^{1 / 6}\right\rangle$ ). Recall that $h_{8}$ counts $\left\langle\Delta_{3}^{1 / 6}, \Delta_{3}^{1 / 6}, \Delta_{3}^{4 / 6}\right\rangle$, whereas $h_{9}$ counts $\left\langle\Delta_{2}^{2 / 3}, \Delta_{3}^{1 / 6}, \Delta_{3}^{1 / 6}\right\rangle$. have

Using the identity $\lambda \cdot \frac{2+\tau}{3}=\frac{2 a-b}{3}+\frac{a+b}{3} \tau$ and proceeding as in case (a), we

$$
\lambda \cdot \frac{2+\tau}{3} \in p^{-1}\left(w_{3}\right) \Longleftrightarrow 3 \mid N\left(=a^{2}-a b+b^{2}\right)
$$

and

$$
\lambda \cdot \frac{2+\tau}{3} \in p^{-1}\left(w_{2}\right) \Longleftrightarrow 3 \nmid N\left(=a^{2}-a b+b^{2}\right) .
$$

It is easy to see that the six rhombi related by $\mathbb{Z}_{6}$-rotation at the origin represent the same map, and the degrees of these rhombi are also given by $|\lambda|^{2}$. By comparing with the decomposition of $F$ in terms of the $q$ th power $(\bmod 3)$ as in Section 5.3, it follows that $h_{8}(q)=\frac{1}{6} F_{0,3}\left(q^{2}\right)$ and $h_{9}(q)=\frac{1}{6} F_{1,3}\left(q^{2}\right)$ if one can prove that there are no other contributions.

\section{CONJECTURE 6.3}

We conjecture that there are no contributions from $\mathbb{P}_{3,6,6}^{1}$ other that these rhombi or, equivalently,

$$
\begin{aligned}
h_{8}(q) & =\frac{1}{6} F_{0,3}\left(q^{2}\right)=\frac{1}{2} f_{1}^{\mathbb{P}_{3,3,3}^{1}}\left(q^{2}\right) \\
& =\frac{1}{6}+q^{6}+q^{18}+q^{24}+2 q^{42}+q^{54}+q^{72}+2 q^{78}+O\left(q^{96}\right), \\
h_{9}(q) & =\frac{1}{6} F_{1,3}\left(q^{2}\right)=f_{0}^{\mathbb{P}_{3,3,3}^{1}}\left(q^{2}\right) \\
& =q^{2}+q^{8}+2 q^{14}+2 q^{26}+q^{32}+2 q^{38}+O\left(q^{48}\right) .
\end{aligned}
$$

REMARK 6.4

One way to see that the conjecture holds true is the following. It can be shown 
that the conjectural answer is modular on $\Gamma(6)$ and the first few terms match with the one given in [MR] (or [MSh]). Then by modularity, they must be identically the same. (Hence, it is not a conjecture in the normal sense.) We left it as a conjecture as the purpose of the article is the direct classification of holomorphic orbispheres, which we failed to show for these particular 3-point invariants.

There is a nontrivial algebraic relation between $h_{8}(q)$ and $h_{9}(q)$ which basically comes from the Frobenius structure on $Q H_{\text {orb }}^{*}\left(\mathbb{P}_{2,3,6}^{1} ; \mathbb{Q}\right)$. This can be obtained as follows. First,

$$
\begin{aligned}
h_{8} & =\left(\Delta_{3}^{1 / 6} * \Delta_{3}^{1 / 6}, \Delta_{3}^{4 / 6}\right) \\
& =\left(\Delta_{3}^{1 / 6} * \Delta_{3}^{1 / 6}, \frac{1}{6}\left(h_{6}\right)^{-1} \Delta_{2}^{1 / 3} * \Delta_{2}^{1 / 3}-\frac{1}{2}\left(h_{6}\right)^{-1} h_{7} \Delta_{2}^{2 / 3}\right) \\
& =-\frac{1}{2}\left(h_{6}\right)^{-1} h_{7} h_{9}+\frac{1}{6}\left(h_{6}\right)^{-1}\left(\Delta_{3}^{1 / 6} * \Delta_{3}^{1 / 6}, \Delta_{2}^{1 / 3} * \Delta_{2}^{1 / 3}\right)
\end{aligned}
$$

where $(\cdot, \cdot)$ is the Poincaré paring and, in the second equality, we used

$$
\Delta_{2}^{1 / 3} * \Delta_{2}^{1 / 3}=6 h_{6} \Delta_{3}^{4 / 6}+3 h_{7} \Delta_{2}^{2 / 3},
$$

which is completely known from cases (b) and (c).

The last term in (6.4) can be computed with the help of the Frobenius structure:

$$
\begin{aligned}
\left(\Delta_{3}^{1 / 6} * \Delta_{3}^{1 / 6}, \Delta_{2}^{1 / 3} * \Delta_{2}^{1 / 3}\right) & =\left(\Delta_{3}^{1 / 6}, \Delta_{3}^{1 / 6} * \Delta_{2}^{1 / 3} * \Delta_{2}^{1 / 3}\right) \\
& =\left(\Delta_{3}^{1 / 6},\left(6 h_{1} \Delta_{3}^{3 / 6}+2 h_{0} \Delta_{1}^{1 / 2}\right) * \Delta_{2}^{1 / 3}\right) \\
& =6 h_{1}\left(\Delta_{3}^{1 / 6}, \Delta_{3}^{3 / 6} * \Delta_{2}^{1 / 3}\right)+2 h_{0}\left(\Delta_{3}^{1 / 6}, \Delta_{1}^{1 / 2} * \Delta_{2}^{1 / 3}\right) \\
& =6\left(h_{1}\right)^{2}+2\left(h_{0}\right)^{2}
\end{aligned}
$$

Plugging it into (6.4), we obtain the relation

$$
6 h_{6} h_{8}=-3 h_{7} h_{9}+6\left(h_{1}\right)^{2}+2\left(h_{0}\right)^{2} .
$$

One can check (6.5) numerically up to a high enough order using Mathematica with our conjectural $h_{8}$ and $h_{9}$.

\section{REMARK 6.5}

A similar kind of lifting argument as in case (b) tells us that the $(3,6,6)$ contribution for $\mathbb{P}_{3,3,3}^{1}$ is equivalent to a certain kind of 4-point Gromov-Witten invariant of $\mathbb{P}_{3,3,3}^{1}$ which counts holomorphic orbispheres $\mathbb{P}_{3,3,3,3}^{1} \rightarrow \mathbb{P}_{3,3,3}^{1}$.

\subsection{The product on $Q H_{\text {orb }}^{*}\left(\mathbb{P}_{2,4,4}^{1}\right)$}

Let $E^{\prime}$ be the elliptic curve associated with the lattice $\mathbb{Z}\langle 1, i\rangle$, where $i=\sqrt{-1}$. (In fact, $E$ and $E^{\prime}$ are isomorphic as symplectic manifolds.) Then the quotient of $E^{\prime}$ by the $\mathbb{Z}_{4}$-action which is generated by $i$-multiplication is the elliptic orbifold projective line $\mathbb{P}_{2,4,4}^{1}=\left[E^{\prime} / \mathbb{Z}_{4}\right]$ with three singular points $w_{1}, w_{2}, w_{3}$. Here, $w_{1}$ is 
the point with the local group isomorphic to $\mathbb{Z}_{2}$, and $w_{2}, w_{3}$ have local groups isomorphic to $\mathbb{Z}_{4}$.

The inertial orbifold $\mathcal{I} \mathbb{P}_{2,4,4}^{1}$ consists of the smooth sector together with a $B \mathbb{Z}_{2}$ and two $B \mathbb{Z}_{4}$ 's. As usual, the $\mathbb{Q}$-basis of $H_{\text {orb }}^{*}\left(\mathbb{P}_{2,4,4}^{1}, \mathbb{Q}\right)$ is taken as

$$
1, \Delta_{1}^{1 / 2}, \Delta_{2}^{1 / 4}, \Delta_{2}^{2 / 4}, \Delta_{2}^{3 / 4}, \Delta_{3}^{1 / 4}, \Delta_{3}^{2 / 4}, \Delta_{3}^{3 / 4},[\mathrm{pt}] \text {. }
$$

Then the cohomology of the smooth sector is given by

$$
H_{\text {orb }}^{0}\left(\mathbb{P}_{2,4,4}^{1}, \mathbb{Q}\right)=\mathbb{Q} \cdot 1, \quad H_{\text {orb }}^{2}\left(\mathbb{P}_{2,4,4}^{1}, \mathbb{Q}\right)=\mathbb{Q} \cdot[\mathrm{pt}]
$$

For twist sectors, $\Delta_{1}^{1 / 2} \in H_{\text {orb }}^{1}\left(\mathbb{P}_{2,4,4}^{1}, \mathbb{Q}\right), \Delta_{k}^{j / 4} \in H_{\text {orb }}^{\frac{2 j}{4}}\left(\mathbb{P}_{2,3,6}^{1}, \mathbb{Q}\right) \quad(j=1,2,3$, $k=2,3)$ are generators supported at singular points $w_{1}, w_{2}, w_{3}$, respectively. In a similar way to the $\mathbb{P}_{2,3,6}^{1}$ case, we classify all the triple orbi-insertions with expected dimension 0 and their domain orbifolds:

(a) $\mathbb{P}_{2,4,4}^{1}:\left\langle\Delta_{1}^{1 / 2}, \Delta_{j}^{1 / 4}, \Delta_{k}^{1 / 4}\right\rangle,\left\langle\Delta_{j}^{2 / 4}, \Delta_{j}^{1 / 4}, \Delta_{k}^{1 / 4}\right\rangle,\left\langle\Delta_{j}^{2 / 4}, \Delta_{k}^{1 / 4}, \Delta_{k}^{1 / 4}\right\rangle$ for $j$, $k=2,3$;

(b) $\mathbb{P}_{2,2}^{1}:\left\langle 1, \Delta_{1}^{1 / 2}, \Delta_{1}^{1 / 2}\right\rangle,\left\langle 1, \Delta_{1}^{1 / 2}, \Delta_{k}^{2 / 4}\right\rangle,\left\langle 1, \Delta_{j}^{2 / 4}, \Delta_{k}^{2 / 4}\right\rangle$ for $j, k=2,3$;

(c) $\mathbb{P}_{4,4}^{1}:\left\langle 1, \Delta_{j}^{1 / 4}, \Delta_{k}^{3 / 4}\right\rangle$ for $j, k=2,3$.

Again, (b) and (c) do not occur because of Lemma 4.1. If we denote $\boldsymbol{t}:=$ $\sum t_{j, i} \Delta_{j}^{i}$, then the genus 0 Gromov-Witten potential of $\mathbb{P}_{2,4,4}^{1}$ is written up to order $t^{3}$ as

$$
\begin{aligned}
F_{0}^{\mathbb{P}_{2,4,4}^{1}}(\boldsymbol{t})= & \frac{1}{2} t_{0}^{2} \log q+\frac{1}{2} t_{0} t_{1, \frac{1}{2}} t_{1, \frac{1}{2}}+\frac{1}{4} t_{0} t_{2, \frac{2}{4}} t_{2, \frac{2}{4}} \\
& +t_{2, \frac{1}{4}} t_{2, \frac{3}{4}}+t_{3, \frac{2}{4}} t_{3, \frac{2}{4}}+t_{3, \frac{1}{4}} t_{3, \frac{3}{4}}+\frac{1}{2} t_{1, \frac{1}{2}}\left(t_{2, \frac{1}{4}}^{2}+t_{3, \frac{1}{4}}^{2}\right) \cdot g_{0}(q) \\
& +t_{1, \frac{1}{2}} t_{2, \frac{1}{4}} t_{3, \frac{1}{4}} \cdot g_{1}(q)+\frac{1}{2}\left(t_{2, \frac{2}{4}} t_{2, \frac{1}{4}}^{2}+t_{3, \frac{2}{4}} t_{3, \frac{1}{4}}^{2}\right) \cdot g_{2}(q) \\
& +\frac{1}{2}\left(t_{2, \frac{2}{4}} t_{3, \frac{1}{4}}^{2}+t_{3, \frac{2}{4}} t_{2, \frac{1}{4}}^{2}\right) \cdot g_{3}(q) \\
& +\left(t_{2, \frac{2}{4}} t_{2, \frac{1}{4}} t_{3, \frac{1}{4}}+t_{3, \frac{2}{4}} t_{3, \frac{1}{4}} t_{2, \frac{1}{4}}\right) \cdot g_{4}(q)+O\left(t^{4}\right)
\end{aligned}
$$

The classification in (a) shows that the domain orbisphere should have the same orbifold structure, that is, the contributions only come from maps $\mathbb{P}_{2,4,4}^{1} \rightarrow$ $\mathbb{P}_{2,4,4}^{1}$. Let $p: \mathbb{C} \rightarrow \mathbb{P}_{2,4,4}^{1}$ be the universal covering which factors through the $\mathbb{Z}_{4}$-quotient map $E^{\prime} \rightarrow \mathbb{P}_{2,4,4}^{1}$. We abuse the notation $p$ for covering maps of both the domain and the target $\mathbb{P}_{2,4,4}^{1}$. From the obvious symmetry between $w_{2}$ and $w_{3}$, we may fix one of the orbi-insertions by $\Delta_{2}^{1 / 4}$ (similar to what we did for the $\mathbb{P}_{3,3,3}^{1}$ case). So, we assume that our holomorphic orbisphere sends $z_{2}$ to $w_{2}$.

As before, any holomorphic orbisphere $u$ that we are considering can be lifted to a linear map $\tilde{u}: z \rightarrow \lambda z$ by Lemmas 4.2 and 4.4 . We set the lattice structure on $\mathbb{C}$ induced by the covering $\mathbb{C} \rightarrow \mathbb{P}_{2,4,4}^{1}$ for both the domain and the target as 
follows:

$$
\begin{aligned}
& p^{-1}\left(w_{1}\right)=\left\{\frac{1}{2}(a+i b) \mid \text { either } a \text { or } b \text { is an odd number, but not both }\right\}, \\
& p^{-1}\left(w_{2}\right)=\mathbb{Z}\langle 1, i\rangle \quad(\ni 0), \\
& p^{-1}\left(w_{3}\right)=\left\{\frac{1}{2}(a+i b) \mid \text { both } a \text { and } b \text { are odd numbers }\right\} .
\end{aligned}
$$

Here, we think of $w_{2}$ as the base point associated with the universal cover $(\mathbb{C}, 0)$ (see Figure 11).

Since we have assumed that the orbifold singular point $z_{2}$ is mapped to $w_{2}$, the lifting $\tilde{u}$ of $u: \mathbb{P}_{2,4,4}^{1} \rightarrow \mathbb{P}_{2,4,4}^{1}$ maps $p^{-1}\left(z_{2}\right)$ to $p^{-1}\left(w_{2}\right)$, fixing the origin. Therefore, $\tilde{u}(z)=\lambda z$ for some $\lambda \in \mathbb{Z}[i]$. As mentioned, the degree of a holomorphic orbisphere $u$ is $|\lambda|^{2}=a^{2}+b^{2}$ if the lifting of $u$ is $\tilde{u}(z)=\lambda z$ with $\lambda=a+b i$. Let $G(q)$ denote the power series

$$
G(q)=\sum_{a, b \in \mathbb{Z}} q^{a^{2}+b^{2}} .
$$

See (A.1) for the first few terms of $G$. Note that if we divide $a^{2}+b^{2}$ by 4 , then 3 cannot appear as a remainder for any $a, b \in \mathbb{Z}$. Thus, we can decompose $G$ into $G=G_{0,4}+G_{1,4}+G_{2,4}$ in accordance with the exponent of $q$ modulo 4 .

We determine the orbi-insertion for each $\lambda=a+b i$ in the same way as before. Note that the right-angled isosceles triangle with vertices $\left\{0, \frac{1}{2}, \frac{1+i}{2}\right\}$ is one of the fundamental domains of the upper hemisphere of $\mathbb{P}_{2,4,4}^{1}$ (see the shaded region in Figure 11).

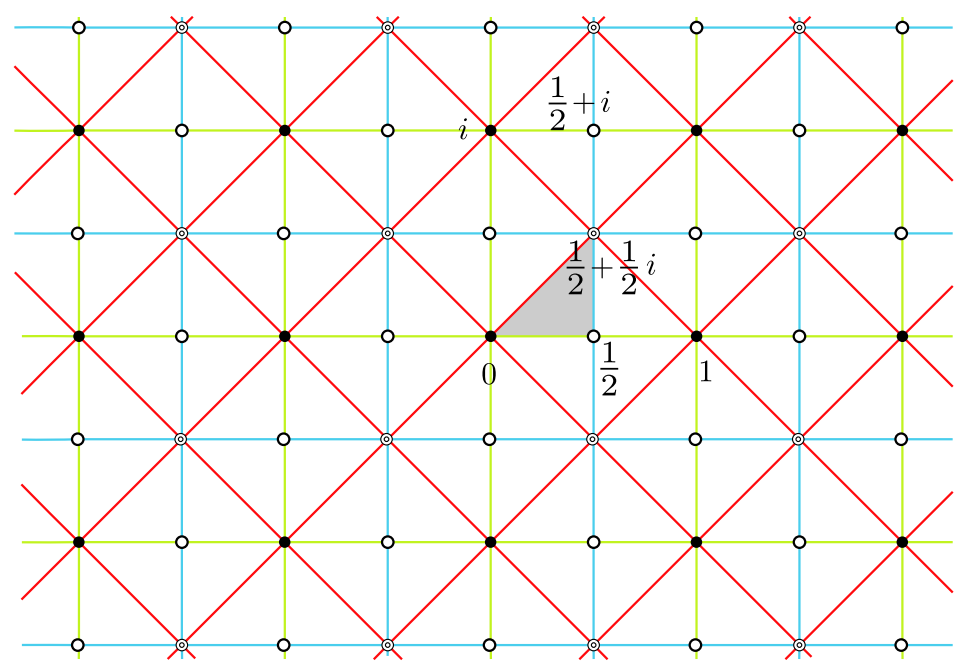

Figure 11. Lattices on the universal cover of $\mathbb{P}_{2,4,4}^{1}: p^{-1}\left(w_{1}\right)=\{\circ\}, p^{-1}\left(w_{2}\right)=\{\bullet\}$, and $p^{-1}\left(w_{3}\right)=\{\odot\}$. 
Observe that the two marked points other than the origin in this fundamental domain map to

$$
(a+b i) \cdot \frac{1}{2}=\frac{a}{2}+\frac{b}{2} i \quad \text { and } \quad(a+b i) \cdot \frac{1+i}{2}=\frac{a-b}{2}+\frac{a+b}{2} i
$$

by the linear map $z \mapsto(a+b i) z$. By proceeding as in the case of $\mathbb{P}_{2,3,6}^{1}$, we see that there are only three possibilities for the types of insertions, which are listed as follows:

(1) " $\frac{a}{2}+\frac{b}{2} \in p^{-1}\left(w_{1}\right)$ and $\frac{a-b}{2}+\frac{a+b}{2} \in p^{-1}\left(w_{3}\right)$ " if and only if $a^{2}+b^{2} \equiv 1$ $\bmod 4$

(2) " $\frac{a}{2}+\frac{b}{2} \in p^{-1}\left(w_{2}\right)$ and $\frac{a-b}{2}+\frac{a+b}{2} \in p^{-1}\left(w_{2}\right)$ " if and only if $a^{2}+b^{2} \equiv 0$ $\bmod 4$;

(3) " $\frac{a}{2}+\frac{b}{2} \in p^{-1}\left(w_{3}\right)$ and $\frac{a-b}{2}+\frac{a+b}{2} \in p^{-1}\left(w_{2}\right)$ " if and only if $a^{2}+b^{2} \equiv 3$ $\bmod 4$.

By the definition of coefficients $g_{i}$ in (6.6), holomorphic orbispheres with insertions (i), (ii), and (iii) precisely give rise to $g_{1}, g_{2}$, and $g_{3}$, respectively. Therefore, we conclude that $g_{0}(q)=g_{4}(q)=0$, and

$$
\begin{aligned}
& g_{1}(q)=\frac{1}{4} \sum_{\substack{N=1 \\
N \equiv 1 \bmod 4}}^{\infty} \sum_{\substack{a^{2}+b^{2}=N \\
a, b \in \mathbb{Z}}} q^{N}=\frac{1}{4} G_{1,4}, \\
& g_{2}(q)=\frac{1}{4} \sum_{\substack{N=1 \\
N \equiv 0 \bmod 4}}^{\infty} \sum_{\substack{a^{2}+b^{2}=N \\
a, b \in \mathbb{Z}}} q^{N}, \\
& g_{3}(q)=\frac{1}{4} \sum_{\substack{N=1 \\
N \equiv 2 \bmod 4}}^{\infty} \sum_{\substack{a^{2}+b^{2}=N \\
a, b \in \mathbb{Z}}}^{\infty} q^{N} .
\end{aligned}
$$

Again, $\frac{1}{4}$ is due to the $\mathbb{Z}_{4}$-symmetry at the origin in the universal cover $\mathbb{C}$ (from the action of the local group at $w_{2}$ ) which is generated by $i$-multiplication.

\section{Appendix A: Theta series}

Recall that our results were expressed in terms of the following two power series:

$$
\begin{aligned}
F(q)= & \sum_{a, b \in \mathbb{Z}} q^{a^{2}-a b+b^{2}} \\
= & 1+6 q+6 q^{3}+6 q^{4}+12 q^{7}+6 q^{9} \\
& +6 q^{12}+12 q^{13}+6 q^{16}+12 q^{19}+12 q^{21}+O\left(q^{24}\right), \\
G(q)= & \sum_{a, b \in \mathbb{Z}} q^{a^{2}+b^{2}} \\
= & 1+4 q+4 q^{2}+4 q^{4}+8 q^{5}+4 q^{8} \\
& +4 q^{9}+8 q^{10}+8 q^{13}+4 q^{16}+8 q^{17}+4 q^{18}+o\left(q^{20}\right) .
\end{aligned}
$$


In this section, we briefly explain several number-theoretic features of $F$ and $G$ (for more details, see [B, Chapter 4] or [G]). We first provide a description of the Fourier coefficients of $F$ and $G$.

PROPOSITION A.1

Write $F(q)=\sum_{N \geq 0} a_{N} q^{N}$, and write $G(q)=\sum_{N \geq 0} b_{N} q^{N}$. Then

$$
\begin{aligned}
& a_{N}=6\left(d_{1 / 3}(N)-d_{2 / 3}(N)\right), \\
& b_{N}=4\left(d_{1 / 4}(N)-d_{3 / 4}(N)\right),
\end{aligned}
$$

where $d_{j / 3}(N)$ denotes the number of divisors of $N$ which are $j$ modulo 3 , and $d_{j / 4}(N)$ denotes the number of divisors of $N$ which are $j$ modulo 4 .

\section{Proof}

We only prove the first identity for $a_{N}$, and we refer the reader to [G, Theorem 3] for $G$. The following is a simple modification of the argument given in [G], but we repeat it here for completeness.

Recall that, for $\tau=e^{2 \pi \sqrt{-1} / 3},|a+b \tau|^{2}=a^{2}-a b+b^{2}$. This gives the structure of a Euclidean domain in $\mathbb{Z}[\tau]$. (This ring is usually called the ring of Eisenstein integers or Eulerian integers.) In particular, $\mathbb{Z}[\tau]$ is a unique factorization domain, and hence, a prime factorization in this ring makes sense up to units which are equal to $\left\{ \pm 1, \pm \tau, \pm \tau^{2}\right\}=\left\{(1+\tau)^{k} \mid 0 \leq k \leq 5\right\}$ (and also up to the order of factors). It is known that a prime number in $\mathbb{Z}[\tau]$ is either a prime number in $\mathbb{Z}$ that is 2 modulo 3 , or $a+b \tau$ whose modulus square $|a+b \tau|^{2}$ is a prime number in $\mathbb{Z}$. In the latter case, $|a+b \tau|^{2}$ is always 1 modulo 3 unless it is $3=(1-\tau) \overline{(1-\tau)}=(1-\tau)(2+\tau)$ itself. (Of course, a prime number multiplied by a unit is also prime.)

Note that finding solutions of

$$
a^{2}-a b+b^{2}=(a+b \tau) \overline{(a+b \tau)}=N \in \mathbb{Z}
$$

is equivalent to finding factorizations $N=\alpha \beta$ of $N$ in $\mathbb{Z}[\tau]$ such that $\beta=\bar{\alpha}$ where $\bar{\alpha}$ is the complex conjugation of $\alpha$. Let $N=3^{f} n_{1} n_{2}$ with $n_{1}=\prod_{p \equiv 1(\bmod 3)} p^{r}$ and $n_{2}=\prod_{q \equiv 2(\bmod 3)} q^{s}$. Then the prime factorization of $N$ in $\mathbb{Z}[\tau]$ can be written as

$$
N=\{(1-\tau)(2+\tau)\}^{f} \prod_{\substack{c^{2}-c d+d^{2}=p \\ p \equiv 1(\bmod 3)}}\{(c+d \tau) \overline{(c+d \tau)}\}^{r} \prod_{q \equiv 2(\bmod 3)} q^{s}
$$

where $c+d \tau$ and $\overline{c+d \tau}$ come in a pair for each $p$ since $N$ is an integer. Now the condition $\beta=\bar{\alpha}$ forces them to be of the following forms:

$$
\begin{aligned}
& \alpha=(1+\tau)^{t}(1-\tau)^{f_{1}}(2+\tau)^{f_{2}} \prod\left\{(c+d \tau)^{r_{1}} \overline{(c+d \tau)}^{r_{2}}\right\} \prod q^{s_{1}}, \\
& \beta=(1+\tau)^{-t}(1-\tau)^{f_{2}}(2+\tau)^{f_{1}} \prod\left\{(c+d \tau)^{r_{2}} \overline{(c+d \tau)}^{r_{1}}\right\} \prod q^{s_{2}}
\end{aligned}
$$

with $0 \leq t \leq 5, f_{1}+f_{2}=f, r_{1}+r_{2}=r$, and $s_{1}+s_{2}=s$. Also $\bar{\beta}=\alpha$ implies that $s_{1}=s_{2}$, so there is no solution to (A.2) if $s$ is odd. Let us assume that $s$ is 
even from now on. Then, the $s_{i}$ 's are uniquely determined (as the halves of $s$ ). Observe that $t$ has six choices, and note that $f_{1}$ and $r_{1}$ determine $f_{2}=f-f_{1}$ and $r_{2}=r-r_{1}$, respectively. Thus, there are seemingly $6(f+1) \prod(r+1)$ choices for $\alpha$ and $\beta$ satisfying (A.3). However, replacing one $(1-\tau)$ by $(2+\tau)$ in the expression of $\alpha$ (A.3) affects $\alpha$ by a unit multiplication since $(2+\tau) /(1-\tau)=1+\tau$ is a multiplicative generator of the group of units in $\mathbb{Z}[\tau]$. Getting rid of this redundancy, the number of pairs $(\alpha, \beta)$ satisfying $N=\alpha \beta$ and $\beta=\bar{\alpha}$ is given by $6 \prod(r+1)$. It is easy to check that this number is the same as $6\left(d_{1 / 3}(N)-\right.$ $\left.d_{2 / 3}(N)\right)$.

\section{REMARK A.2}

From the proof, we see that 6 in the expression of $a_{N}$ is related to the number of units in the ring $\mathbb{Z}[\tau]$, which give the symmetries on the associated moduli space of orbispheres (see the last paragraph of Section 5.2).

We next describe $F$ and $G$ in terms of the famous Jacobi theta functions. The definitions of the related Jacobi theta functions are given as follows.

\section{DEFINITION A.3}

The second and third Jacobi theta functions are the power series $\theta_{2}$ and $\theta_{3}$ in $q$ which are defined as follows:

$$
\begin{aligned}
\theta_{2}(q) & :=\sum_{-\infty}^{\infty} q^{(n+1 / 2)^{2}}, \\
\theta_{3}(q) & :=\sum_{-\infty}^{\infty} q^{n^{2}}
\end{aligned}
$$

\section{REMARK A.4}

Originally, theta functions are two variable functions depending on $z$ and $q$. Above, $\theta_{i}$ is indeed obtained by putting $z=0$.

Let us now express $F(q)$ and $G(q)$ in terms of the $\theta_{i}$ 's $(i=2,3)$. First, for $F$, observe that the number of integer solutions of $x^{2}-x y+y^{2}=N$ is equivalent to that of solutions of $\left(m^{2}+3 n^{2}\right) / 4=N$. To see this, simply put $m=x+y$ and $n=x-y$ into $\left(m^{2}+3 n^{2}\right) / 4$. Note that $m$ and $n$ should have the same parity.

Therefore,

$$
\begin{aligned}
F(q) & =\sum_{x, y \in \mathbb{Z}} q^{x^{2}-x y+y^{2}} \\
& =\sum_{m, n: \text { even }} q^{\frac{m^{2}+3 n^{2}}{4}}+\sum_{m, n: \text { odd }} q^{\frac{m^{2}+3 n^{2}}{4}} \\
& =\sum_{k, l \in \mathbb{Z}} q^{k^{2}+3 l^{2}}+\sum_{k, l \in \mathbb{Z}} q^{((k+1) / 2)^{2}+3((l+1) / 2)^{2}}
\end{aligned}
$$




$$
=\theta_{3}(q) \theta_{3}\left(q^{3}\right)+\theta_{2}(q) \theta_{2}\left(q^{3}\right) .
$$

The expression of $G(q)$ is even simpler since

$$
G(q)=\sum_{x, y \in \mathbb{Z}} q^{x^{2}+y^{2}}=\left(\theta_{3}(q)\right)^{2} .
$$

In general, the theta function associated with a binary quadratic form $Q(x, y)=a x^{2}+b x y+c z^{2}$ is defined by

$$
\theta_{Q}(z)=\sum_{(x, y) \in \mathbb{Z}^{2}} \exp (2 \pi i z Q(x, y)),
$$

where we have used the substitution $q=\exp (2 \pi i z)$ mostly in this article. In the Fourier expansion

$$
\theta_{Q}(z)=\sum_{N=0}^{\infty} R_{Q}(N) \exp (2 \pi i N z)
$$

the numbers $R_{Q}(N)$ are called the representation numbers of the form $Q$, and hence $a_{N}$ and $b_{N}$ above are given as $R_{F}(N)$ and $R_{G}(N)$, respectively.

These theta functions are known to be modular forms of weight 1 on (an appropriately defined subgroup of) the modular group. We believe that this modularity of $F$ and $G$ may help to compare our results with those given in [ST] and [MR].

Acknowledgment. We would like to express our gratitude to our advisor CheolHyun Cho for suggesting the problem and also for valuable discussions. We also thank Yefeng Shen for pointing out certain inaccuracies in an earlier version of this article and explaining his work. The first author thanks Atsushi Takahashi for valuable discussions on the modularity of Gromov-Witten potentials, and Jangwon Ju for an explanation on theta series. We thank an anonymous referee for suggestions that improved the exposition of this article.

\section{References}

[ALR] A. Adem, J. Leida, and Y. Ruan, Orbifolds and Stringy Topology, Cambridge Tracts in Math. 171, Cambridge Univ. Press, Cambridge, 2007. MR 2359514. DOI 10.1017/CBO9780511543081.

[B] E. Bannai, Sphere Packings, Lattices and Groups, 3rd ed., Grundlehren Math. Wiss. 290, Springer, New York, 1999. MR 1662447. DOI 10.1007/978-1-4757-6568-7.

[CR] W. Chen and Y. Ruan, "Orbifold Gromov-Witten theory" in Orbifolds in Mathematics and Physics (Madison, WI, 2001), Contemp. Math. 310, Amer. Math. Soc., Providence, 2002, 25-85. MR 1950941. DOI $10.1090 /$ conm/310/05398.

[CR1] A new cohomology theory of orbifold, Comm. Math. Phys. 248 (2004), 1-31. MR 2104605. DOI 10.1007/s00220-004-1089-4. 
[CHKL] C.-H. Cho, H. Hong, S. Kim, and S.-C. Lau, Lagrangian Floer potentials of orbifold spheres, preprint, arXiv:1403.0990v1 [math.SG].

[D] M. W. Davis, "Lectures on orbifolds and reflection groups" in Transformation Groups and Moduli Spaces of Curves, Adv. Lect. Math. (ALM) 16, Int. Press, Somerville, Mass., 2011, 63-93. MR 2883685.

[G] E. Grosswald, Representations of Integers as Sums of Squares, Springer, New York, 1985. MR 0803155. DOI 10.1007/978-1-4613-8566-0.

[KS] M. Krawitz and Y. Shen, Landau-Ginzburg/Calabi-Yau correspondence of all genera for elliptic orbifold $\mathbb{P}^{1}$, preprint, arXiv:1106.6270v1 [math.AG].

[McS] D. McDuff and D. Salamon, J-Holomorphic Curves and Quantum Cohomology, Univ. Lecture Ser. 6, Amer. Math. Soc., Providence, 1994. MR 1286255. DOI 10.1090/ulect/006.

[MR] T. E. Milanov and Y. Ruan, Gromov-Witten theory of elliptic orbifold $P^{1}$ and quasi-modular forms, preprint, arXiv:1106.2321v1 [math.AG].

[MSh] T. E. Milanov and Y. Shen, Global mirror symmetry for invertible simple elliptic singularities, preprint, arXiv:1210.6862v2 [math.AG].

[MT] T. E. Milanov and H.-H. Tseng, The spaces of Laurent polynomials, Gromov-Witten theory of $\mathbb{P}^{1}$-orbifolds, and integrable hierarchies, J. Reine Angew. Math. 622 (2008), 189-235. MR 2433616.

DOI 10.1515/CRELLE.2008.069.

[R] P. Rossi, Gromov-Witten theory of orbicurves, the space of tri-polynomials and symplectic field theory of Seifert fibrations, Math. Ann. 348 (2010), 265-287. MR 2672302. DOI 10.1007/s00208-009-0471-0.

[S] K. Saito, Duality for regular systems of weights, Asian J. Math. 2 (1998), 983-1047. MR 1734136.

[Sa] I. Satake, On a generalization of the notion of manifold, Proc. Natl. Acad. Sci. USA 42 (1956), 359-363. MR 0079769.

[ST] I. Satake and A. Takahashi, Gromov-Witten invariants for mirror orbifolds of simple elliptic singularities, Ann. Inst. Fourier (Grenoble) 61 (2011), 2885-2907. MR 3112511.

[T] Y. Takeuchi, Waldhausen's classification theorem for finitely uniformizable 3-orbifolds, Trans. Amer. Math. Soc. 328, no. 1 (1991), 151-200.

MR 1065604. DOI 10.2307/2001880.

[Thu] W. P. Thurston, The geometry and topology of three-manifolds, Princeton lecture notes, 1979, http://library.msri.org/books/gt3m/.

Hong: Department of Mathematics/Institute of Mathematical Sciences, Chinese University of Hong Kong, Shatin, New Territories, Hong Kong;

hhong@ims.cuhk.edu.hk; hansol84@gmail.com

Shin: School of Mathematics, Korea Institute for Advanced Study, Seoul, Republic of Korea; hsshin@kias.re.kr 